\title{
ON THE SOLVABILITY OF THE NEUMANN PROBLEM IN DOMAINS WITH PEAK
}

\author{
V. G. MAZ'YA AND S. V. POBORCHII
}

Dedicated to V. P. Havin on the occasion of his 75th birthday

\begin{abstract}
The Neumann problem is considered for a quasilinear elliptic equation of second order in a multidimensional domain with the vertex of an isolated peak on the boundary. Under certain assumptions, the study of the solvability of this problem is reduced to a description of the dual to the Sobolev space $W_{p}^{1}(\Omega)$ or (in the case of a homogeneous equation with nonhomogeneous boundary condition) to the boundary trace space $T W_{p}^{1}(\Omega)$. This description involves Sobolev classes with negative smoothness exponent on Lipschitz domains or Lipschitz surfaces, and also some weighted classes of functions on the interval $(0,1)$ of the real line. The main results are proved on the basis of the known explicit description of the spaces $T W_{p}^{1}(\Omega)$ on a domain with an outward or inward cusp on the boundary.
\end{abstract}

\section{INTRODUCTION}

We consider the Neumann problem for a quasilinear second order elliptic equation in a multidimensional domain $\Omega$. Under certain conditions, the study of its solvability is reduced to a description of the space $W_{p}^{1}(\Omega)^{*}$ dual to the Sobolev space $W_{p}^{1}(\Omega)$, or to a description of the space $T W_{p}^{1}(\Omega)^{*}$ dual to the space of boundary traces of the functions in $W_{p}^{1}(\Omega)$. In the present paper we give an explicit characterization of the spaces $W_{p}^{1}(\Omega)^{*}$ and $T W_{p}^{1}(\Omega)^{*}$ for a domain with the vertex of an isolated cusp on the boundary.

The paper consists of five sections. In $\S 1$ we state the Neumann problem and relate its solvability to the description of the conjugate spaces mentioned above. In fact, $\S 1$ is a detailed introduction. In $\S 2$ we prove Theorem 1, which characterizes the space $W_{p}^{1}(\Omega)^{*}$ for domains with an outward cusp. In $\S 3$ we establish some lemmas to be used in the sequel. A description of the space $T W_{p}^{1}(\Omega)^{*}$ for the domain with an outward cusp is obtained in $\S 4$ (Theorem 2). This space is characterized in terms of the classes $\left(W_{p}^{1-1 / p}(S)\right)^{*}$ on Lipschitz surfaces, and in terms of some function spaces on the interval $(0,1)$ of the real axis.

The proof of Theorem 2 is based on an explicit description of the trace space $T W_{p}^{1}(\Omega)$; see [7] and [8, 7.2]. One of the consequences of Theorem 2 is a necessary and sufficient condition on the boundary function of class $L_{q}(\partial \Omega)$ ensuring the unique solvability of the Neumann problem.

In the final $\S 5$ we deal with domains having the vertex of an isolated inward cusp on the boundary. The principle results of the paper are stated in Theorem $1(\S 2)$, Theorem 2 (§4), and Theorem $3(\S 5)$.

2000 Mathematics Subject Classification. Primary 35J25.

Key words and phrases. Neumann problem, Sobolev spaces, domains with cusps, boundary traces, dual spaces.

The research of the second author was supported by RFBR (grant no. 08-01-00676-a). 


\section{Preliminaries}

We begin with the following simple general observation.

Lemma 1. Let $X$ be a Banach space and $X_{0}$ its closed subspace. Let $\dot{X}$ denote the quotient space $X / X_{0}$ with the norm

$$
\|\dot{x}\|=\inf \{\|y\|: y \in \dot{x}\} .
$$

(i) If $f \in X^{*}$ (i.e., $f$ is a continuous linear functional on $X$ ) and $\left.f\right|_{X_{0}}=0$, then the functional $\dot{f}$ defined by

$$
\dot{X} \ni \dot{x} \mapsto \dot{f}(\dot{x})=f(x)
$$

belongs to $(\dot{X})^{*}$, and $\|\dot{f}\|=\|f\|$.

(ii) If $\dot{f} \in(\dot{X})^{*}$, then the functional

$$
X \ni x \mapsto f(x)=\dot{f}(\dot{x})
$$

is in $X^{*}$, and $\left.f\right|_{X_{0}}=0$.

The proof of this assertion follows easily from the definition of the quotient space.

Let $\Omega$ be a domain in $\mathbb{R}^{n}$, and let $p \in(1, \infty)$. By $W_{p}^{1}(\Omega)$ we mean the Sobolev space of functions on $\Omega$ characterized by the finiteness of the norm

$$
\|u\|_{W_{p}^{1}(\Omega)}=\left(\|u\|_{L_{p}(\Omega)}^{p}+\|\nabla u\|_{L_{p}(\Omega)}^{p}\right)^{1 / p} .
$$

We also introduce the space $T W_{p}^{1}(\Omega)$ consisting of the traces $\left.u\right|_{\partial \Omega}$ of all functions in $W_{p}^{1}(\Omega)$. This space is endowed with the norm

$$
\|v\|_{T W_{p}^{1}(\Omega)}=\inf \left\{\|u\|_{W_{p}^{1}(\Omega)}:\left.u\right|_{\partial \Omega}=v\right\} .
$$

If the boundary of $\Omega$ is "sufficiently nice", then the trace space $T W_{p}^{1}(\Omega)$ admits an explicit description. In accordance with Gagliardo's theorem [1, for domains of class $C^{0,1}$ (i.e., for domains with compact closure whose boundary is locally a Lipschitz graph), $T W_{p}^{1}(\Omega)$ coincides with the space $W_{p}^{1-1 / p}(S), S=\partial \Omega$, consisting of all functions on $S$ with finite norm

where

$$
\|v\|_{W_{p}^{1-1 / p}(S)}=\|v\|_{L_{p}(S)}+[v]_{p, S}
$$

$$
[v]_{p, S}=\left(\iint_{S \times S}|v(x)-v(\xi)|^{p} \frac{d s_{x} d s_{\xi}}{|x-\xi|^{n+p-2}}\right)^{1 / p},
$$

and $d s_{x}, d s_{\xi}$ are the area elements on $S$.

For general domains we can put $T W_{p}^{1}(\Omega)=W_{p}^{1}(\Omega) / \stackrel{\circ}{W}_{p}^{1}(\Omega)$, where $\stackrel{\circ}{W}_{p}^{1}(\Omega)$ denotes the closure in $W_{p}^{1}(\Omega)$ of the set $C_{0}^{\infty}(\Omega)$ of all smooth functions compactly supported in $\Omega$.

Suppose the boundary of a domain $\Omega$ is compact and has finitely many non-Lipschitz points (e.g., $\Omega$ is a domain with the vertex of an isolated cusp on $\partial \Omega$ ). Then a normal to $\partial \Omega$ exists almost everywhere with respect to the $(n-1)$-dimensional area. Suppose that the area of $\partial \Omega$ is finite and consider the Neumann problen 1

$$
\begin{aligned}
& -\operatorname{div}\left(|\nabla u|^{p-2} \nabla u\right)+a|u|^{p-2} u=0 \quad \text { on } \Omega, \\
& \left.|\nabla u|^{p-2} \frac{\partial u}{\partial \nu}\right|_{\partial \Omega}=f,
\end{aligned}
$$

\footnotetext{
${ }^{1}$ We consider the model problem (1.2), (1.3) for simplicity of presentation. In fact, all results below are true for elliptic equations of a more general type [2].
} 
where $p \in(1, \infty), \nu$ is the unit outward normal to $\partial \Omega$ at a boundary point, $a \in L_{\infty}(\Omega)$, $a(x) \geq$ const $>0$ a.e. on $\Omega$, and $f$ is a linear functional defined on the set $\mathcal{V}=$ $W_{p}^{1}(\Omega) \cap L_{\infty}(\Omega) \cap C^{\infty}(\Omega)$ and vanishing on $C_{0}^{\infty}(\Omega)$.

A function $u \in W_{p}^{1}(\Omega)$ is called the solution of problem (1.2), (1.3) if the identity

$$
L(u, v)=\langle f, v\rangle
$$

is fulfilled for all $v \in \mathcal{V}$, where $\langle f, v\rangle$ is the value of $f$ at $v$, and

$$
L(u, v)=\int_{\Omega}\left(|\nabla u|^{p-2} \nabla u \cdot \nabla v+a|u|^{p-2} u v\right) d x .
$$

We observe that, for fixed $u \in W_{p}^{1}(\Omega)$, the left-hand side of (1.4) is a continuous linear functional with respect to $v \in W_{p}^{1}(\Omega)$, and the set $\mathcal{V}$ is dense in $W_{p}^{1}(\Omega)$; see [5, 3.1.2]. Therefore, (1.4) implies that $f$ extends uniquely to a functional in $W_{p}^{1}(\Omega)^{*}$ vanishing for $v \in \stackrel{\circ}{W}_{p}^{1}(\Omega)$, and hence lying in $T W_{p}^{1}(\Omega)^{*}$ by Lemma 1 .

Suppose that $F$ is a linear functional on $\mathcal{V}$ (possibly, $\operatorname{Ker} F \not \supset C_{0}^{\infty}(\Omega)$ ). Along with (1.4), we consider yet another problem of finding $u \in W_{p}^{1}(\Omega)$ such that

$$
L(u, v)=\langle F, v\rangle
$$

for all $v \in \mathcal{V}$. If the latter problem is solvable, then, again, $F$ extends uniquely to a functional in $W_{p}^{1}(\Omega)^{*}$.

Now we show that for any $F \in W_{p}^{1}(\Omega)^{*}$ (possibly, $\left.F \notin T W_{p}^{1}(\Omega)^{*}\right)$ there is a unique function $u \in W_{p}^{1}(\Omega)$ satisfying (1.5) for all $v \in W_{p}^{1}(\Omega)$. Indeed, if we assume that problem (1.5) has two solutions $\left(u_{1}\right.$ and $\left.u_{2}\right)$, then (1.5) implies that

$$
L\left(u_{1}, u_{1}-u_{2}\right)-L\left(u_{2}, u_{1}-u_{2}\right)=0
$$

or

$$
\begin{aligned}
& \int_{\Omega}\left(\left|\nabla u_{1}\right|^{p-2} \nabla u_{1}-\left|\nabla u_{2}\right|^{p-2} \nabla u_{2}\right)\left(\nabla u_{1}-\nabla u_{2}\right) d x \\
& \quad+\int_{\Omega} a\left(\left|u_{1}\right|^{p-2} u_{1}-\left|u_{2}\right|^{p-2} u_{2}\right)\left(u_{1}-u_{2}\right) d x=0 .
\end{aligned}
$$

Since the function $\mathbb{R}^{n} \ni \xi \mapsto \psi(\xi)=|\xi|^{p}$ is strictly convex for $p>1$, it follows that $(\nabla \psi(\xi)-\nabla \psi(\eta))(\xi-\eta)>0$ for $\xi \neq \eta$, i.e.,

$$
\left(|\xi|^{p-2} \xi-|\eta|^{p-2} \eta\right)(\xi-\eta)>0, \quad \xi \neq \eta
$$

Relations (1.7) and (1.6) imply that the integrands in (1.6) vanish almost everywhere on $\Omega$. Then $u_{1}=u_{2}$ almost everywhere, by (1.7). So there is at most one element $u \in W_{p}^{1}(\Omega)$ satisfying $(1.5)$.

We prove that problem (1.5) is solvable. Note that the expression $p L(u, v)-p\langle F, v\rangle$ is the variation of the functional

$$
W_{p}^{1}(\Omega) \ni v \mapsto G(v)=\int_{\Omega}\left(|\nabla v|^{p}+a|v|^{p}\right) d x-p\langle F, v\rangle .
$$

Therefore, (1.5) is true for all $v \in W_{p}^{1}(\Omega)$ if $u$ provides the minimum to the functional $G$ on $W_{p}^{1}(\Omega)$. We check that the minimum is attained. For this, we consider a minimizing sequence $\left\{v_{k}\right\}$ for $G$. Since $F$ is continuous on $W_{p}^{1}(\Omega)$, there are positive constants $c_{1}, c_{2}>0$ such that

$$
G(v) \geq c_{1}\|v\|_{W_{p}^{1}(\Omega)}^{p}-c_{2}\|v\|_{W_{p}^{1}(\Omega)} .
$$


Since $p>1$, we have $G(v) \rightarrow+\infty$ as $\|v\|_{W_{p}^{1}(\Omega)} \rightarrow \infty$. This means that the above minimizing sequence is bounded and, hence, has a subsequence (which we relabel as $\left\{v_{k}\right\}$ ) weakly convergent in $W_{p}^{1}(\Omega)$. Let $u$ be its limit. We claim that

$$
G(u)=\min \left\{G(v): v \in W_{p}^{1}(\Omega)\right\} .
$$

Clearly, the norm

$$
[v]=\left(\int_{\Omega}\left(|\nabla v|^{p}+a|v|^{p}\right) d x\right)^{1 / p}
$$

is equivalent to $\|v\|_{W_{p}^{1}(\Omega)}$. For any $\varepsilon>0$ there is a functional $f_{0} \in W_{p}^{1}(\Omega)^{*}$ whose norm with respect to the new norm in $W_{p}^{1}(\Omega)$ equals 1 and

$$
[u]^{p}-\varepsilon<\left|\left\langle f_{0}, u\right\rangle\right|^{p}=\lim _{k \rightarrow \infty}\left|\left\langle f_{0}, v_{k}\right\rangle\right|^{p} .
$$

Then $\left[v_{k}\right]^{p}>[u]^{p}-\varepsilon$ for all sufficiently large $k$. For the same $k$ we have

$$
G\left(v_{k}\right)>[u]^{p}-\varepsilon-p\left\langle F, v_{k}\right\rangle .
$$

Since $\left\langle F, v_{k}\right\rangle \rightarrow\langle F, u\rangle$, it follows that

$$
\inf \left\{G(v): v \in W_{p}^{1}(\Omega)\right\} \geq[u]^{p}-\varepsilon-p\langle F, u\rangle
$$

and since $\varepsilon>0$ is arbitrary, we obtain (1.8).

We have proved the following assertion: if the functional on the right in (1.5) is continuous on $W_{p}^{1}(\Omega)$, then (1.5) holds true for a unique $u \in W_{p}^{1}(\Omega)$ and all $v \in W_{p}^{1}(\Omega)$. Thus, the study of the solvability of problem (1.2), (1.3) (or equivalently, problem (1.4) with the functional $f$ vanishing on $C_{0}^{\infty}(\Omega)$ ), and also the study of the solvability of the more general problem (1.5), reduces to a description of the spaces $T W_{p}^{1}(\Omega)^{*}$ and $W_{p}^{1}(\Omega)^{*}$, respectively. Below we give an explicit description of these spaces for a domain with the vertex of an isolated peak on the boundary.

In what follows, for domains $\Omega \in C^{0,1}$, we write $W_{p^{\prime}}^{-1}(\Omega)$ instead of $W_{p}^{1}(\Omega)^{*}$, where $p^{\prime}=p /(p-1)$, and we define

$$
\|f\|_{W_{p^{\prime}}^{-1}(\Omega)}=\sup \left\{|\langle f, v\rangle|: v \in W_{p}^{1}(\Omega),\|v\|_{W_{p}^{1}(\Omega)}=1\right\} .
$$

Also, if $S$ is a Lipschitz surface, we write $W_{p^{\prime}}^{-1 / p^{\prime}}(S)$ instead of $\left(W_{p}^{1-1 / p}(S)\right)^{*}$, and the norm in this space is

$$
\|f\|_{W_{p^{\prime}}^{-1 / p^{\prime}}(S)}=\sup \left\{|\langle f, v\rangle|: v \in W_{p}^{1-1 / p}(S),\|v\|=1\right\} .
$$

For a domain with outward peak, the space $W_{p}^{1}(\Omega)^{*}$ is characterized in terms of the spaces $W_{p^{\prime}}^{-1}$ for domains of class $C^{0,1}$, and in terms of some function spaces on the interval $(0,1)$ of the real axis. In a similar way, for a domain with outward or inward peak, the space $\left(T W_{p}^{1}(\Omega)\right)^{*}$ is characterized in terms of the classes $W_{p^{\prime}}^{-1 / p^{\prime}}$ for Lipschitz surfaces and in terms of some function spaces on the interval $(0,1)$.

\section{$\S 2$. The SPACE $W_{p}^{1}(\Omega)^{*}$ FOR A DOMAIN With OUTWARD PEAK}

First, we define a domain with outward peak. Let $\Omega$ be a bounded domain in $\mathbb{R}^{n}$ $(n>2)$, let $O \in \partial \Omega$, and suppose that the surface $\partial \Omega \backslash\{O\}$ is locally a Lipschitz graph. At $O$ we locate the origin of the Cartesian coordinates $x=(y, z), y \in \mathbb{R}^{n-1}, z \in \mathbb{R}^{1}$. Let $\varphi$ be a monotone increasing function of class $C^{0,1}([0,1])$ such that $\varphi(0)=0, \varphi^{\prime}(t) \rightarrow 0$ as $t \rightarrow+0$, and let $\omega$ be a bounded domain in $\mathbb{R}^{n-1}$ of class $C^{0,1}$. 
Definition 1. The point $O$ is called the vertex of a peak directed into the exterior of $\Omega$ if there is a neighborhood $U$ of this point for which

$$
U \cap \Omega=\{x=(y, z): z \in(0,1), y / \varphi(z) \in \omega\} .
$$

For simplicity of presentation, in what follows we assume that

$$
\partial \Omega \cap U=\{x: 0<z<1, y / \varphi(z) \in \partial \omega\} .
$$

We introduce some notation. If $F \in W_{p}^{1}(\Omega)^{*}$ and $\lambda$ is a Lipschitz function on $\Omega$, we set

$$
\langle\lambda F, v\rangle=\langle F, \lambda v\rangle, \quad v \in W_{p}^{1}(\Omega) .
$$

Let $v$ be a function defined on $\Omega \cap U$. The mean value of $v$ on the section of $\Omega$ by the hyperplane $z=$ const is defined as

$$
\widetilde{v}(z)=\frac{1}{|\omega|} \int_{\omega} v(\varphi(z) y, z) d y
$$

where $|\omega|=\operatorname{mes}_{n-1}(\omega)$.

If $F \in W_{p}^{1}(\Omega)^{*}$ and the support of $v \in W_{p}^{1}(\Omega)$ belongs to $\Omega \cap U$, then, by definition, we put $\langle\widetilde{F}, v\rangle=\langle F, \widetilde{v}\rangle$.

A special partition of unity on $\Omega$ will play an important role in the sequel. To construct it, first we introduce a sequence $\left\{z_{k}\right\}$ by

$$
z_{0} \in(0,1), \quad z_{k+1}+\varphi\left(z_{k+1}\right)=z_{k}, \quad k=0,1, \ldots
$$

Clearly, $\left\{z_{k}\right\}$ is monotone decreasing, and furthermore,

$$
z_{k} \rightarrow 0, \quad z_{k+1}^{-1} z_{k} \rightarrow 1, \quad \varphi\left(z_{k+1}\right)^{-1} \varphi\left(z_{k}\right) \rightarrow 1 .
$$

Consider a smooth partition of unity $\left\{\mu_{k}\right\}_{k \geq 1}$ subordinate to the covering of $\left(0, z_{1}\right]$ by the intervals $\Delta_{k}=\left(z_{k+1}, z_{k-1}\right)$, i.e., a set of functions $\mu_{k} \in C_{0}^{\infty}\left(\Delta_{k}\right)$ satisfying

$$
0 \leq \mu_{k} \leq 1, \quad \sum_{k \geq 1} \mu_{k}(z)=1, \quad z \in\left(0, z_{1}\right]
$$

Such a partition can be constructed in such a way that

$$
\operatorname{dist}\left(\operatorname{supp} \mu_{k}, \mathbb{R}^{1} \backslash \Delta_{k}\right) \geq \text { const } \cdot \varphi\left(z_{k}\right), \quad\left|\mu_{k}^{\prime}\right| \leq \text { const } \cdot \varphi\left(z_{k}\right)^{-1}
$$

with positive constants depending only on $\varphi$ and $z_{0}$, and the identity $\sum_{k \geq 1} \mu_{k}(z)=1$ is fulfilled for $z \in(0, \delta]$ with some $\delta>z_{1}$.

Let $\left\{\lambda_{k}\right\}_{k \geq 1}$ be yet another set of functions subject to

$$
0 \leq \lambda_{k} \leq 1, \quad \lambda_{k} \in C_{0}^{\infty}\left(\Delta_{k}\right),\left.\quad \lambda_{k}\right|_{\operatorname{supp} \mu_{k}}=1 .
$$

Then $\lambda_{k} \mu_{k}=\mu_{k}$ for all $k \geq 1$. We put $\mu_{0}(z)=0$ for $z<z_{1}$ and $\mu_{0}(z)=1-\mu_{1}(z)$ for $z \geq z_{1}$. Then, obviously, $\sum_{k \geq 0} \mu_{k}(z)=1$ for all $z \in(0,1]$. The partition of unity constructed in this way and the set of functions $\left\{\lambda_{k}\right\}$ depend only on $\varphi$ and $z_{0}$. We view them as fixed and do not mention the dependence on them of positive constants appearing below.

We also introduce the "cells"

$$
\Omega_{k}=\left\{(y, z) \in \Omega \cap U: z \in \Delta_{k}\right\}, \quad \Delta_{k}=\left(z_{k+1}, z_{k-1}\right), \quad k=1,2, \ldots,
$$

and put

$$
\Omega_{0}=\Omega \backslash\left\{x \in \Omega \cap U: z \leq z_{1}\right\} .
$$

We note that the partition of unity constructed above for the interval $(0,1]$ induces a partition of unity for $\Omega$ if we define $\mu_{0}=1$ on $\Omega_{0} \backslash(\Omega \cap U)$ and $\mu_{k}(x)=\mu_{k}(z)$ for $x \in \Omega \cap U, k \geq 0$, and define $\mu_{k}=0$ on $\Omega \backslash U$ for $k \geq 1$. This partition of unity for $\Omega$ is subordinate to the covering $\left\{\Omega_{k}\right\}_{k \geq 0}$ in the sense that $\mu_{k}=0$ on $\Omega \backslash \Omega_{k}$. 
We say that the support of a functional $F \in W_{p}^{1}(\Omega)^{*}$ lies in $\Omega_{k}$ (and write $\operatorname{supp} F \subset$ $\left.\Omega_{k}\right)$ if $\left.v\right|_{\Omega_{k}}=0$ implies $\langle F, v\rangle=0$.

In what follows we shall need some estimates for the mean value (2.2).

Lemma 2. For $v \in L_{p}(\Omega \cap U)$ we have

$$
\int_{a}^{b}|\widetilde{v}(z)|^{p} \varphi(z)^{n-1} d z \leq c \int_{a}^{b} d z \int_{\{y / \varphi(z) \in \omega\}}|v(y, z)|^{p} d y,
$$

and for $v \in W_{p}^{1}(\Omega \cap U)$ we have

$$
\int_{a}^{b}\left|\widetilde{v}^{\prime}(z)\right|^{p} \varphi(z)^{n-1} d z \leq c \int_{a}^{b} d z \int_{\{y / \varphi(z) \in \omega\}}|\nabla v(y, z)|^{p} d y,
$$

where $0 \leq a<b \leq 1$ and the constants $c$ do not depend on $a, b, v$.

Proof. The first estimate is a simple consequence of Hölder's inequality.

Turning to (2.4), we show that the function $(0,1) \ni z \mapsto \widetilde{v}(z)$ is absolutely continuous and estimate its derivative. Note that, for any $\varepsilon \in(0,1)$, the function

$$
\omega \times(\varepsilon, 1) \ni(y, z) \mapsto w(y, z)=v(\varphi(z) y, z)
$$

belongs to the class $W_{p}^{1}(\omega \times(\varepsilon, 1))$. Hence, $w(y, \cdot)$ is an absolutely continuous function for almost all $y \in \omega$. Therefore, for any $\eta \in C_{0}^{\infty}(0,1)$ we obtain

$$
\int_{0}^{1} \widetilde{v}(z) \eta^{\prime}(z) d z=-\frac{1}{|\omega|} \int_{\omega} d y \int_{0}^{1} \frac{\partial w}{\partial z}(y, z) \eta(z) d z .
$$

So, $\widetilde{v}$ is absolutely continuous on the interval $(0,1)$, and

$$
\widetilde{v}^{\prime}(z)=\frac{1}{|\omega|} \int_{\omega}\left(\frac{\partial v}{\partial z}(\varphi(z) y, z)+\sum_{i=1}^{n-1} \frac{\partial v}{\partial y_{i}}(\varphi(z) y, z) \varphi^{\prime}(z) y_{i}\right) d y
$$

for almost all $z \in(0,1)$. Applying the Hölder inequality, we arrive at the estimates

$$
\begin{aligned}
& \int_{a}^{b}\left|\widetilde{v}^{\prime}(z)\right|^{p} \varphi(z)^{n-1} d z \\
& \quad \leq \frac{1}{|\omega|} \int_{a}^{b} \varphi(z)^{n-1} d z \int_{\omega}\left|\frac{\partial v}{\partial z}(\varphi(z) y, z)+\sum_{i=1}^{n-1} \frac{\partial v}{\partial y_{i}}(\varphi(z) y, z) \varphi^{\prime}(z) y_{i}\right|^{p} d y \\
& \quad \leq c \int_{a}^{b} d z \int_{\varphi(z) \omega}|\nabla v(y, z)|^{p} d y \leq c\|\nabla v\|_{L_{p}(\Omega)}^{p},
\end{aligned}
$$

which concludes the proof of the lemma.

The theorem stated below gives a description of the space $W_{p}^{1}(\Omega)^{*}$ dual to $W_{p}^{1}(\Omega)$, for a domain with outward peak. As a preliminary, we introduce the space $X_{p}(0,1)$ of functions in $L_{p, \text { loc }}(0,1)$ with finite norm

$$
\|u\|_{X_{p}(0,1)}=\left(\int_{0}^{1}\left(|u(z)|^{p}+\left|u^{\prime}(z)\right|^{p}\right) \varphi(z)^{n-1} d z\right)^{1 / p} .
$$

Theorem 1. Let $\Omega$ be a domain with outward peak, and let $\left\{\mu_{k}\right\}_{k \geq 0}$ be the partition of unity for $\Omega$ constructed above.

(i) Let $F \in W_{p}^{1}(\Omega)^{*}$. Then $F$ can be written as the sum of three terms

$$
F=\mu_{0} F+\left(1-\mu_{0}\right) \widetilde{F}+\left(1-\mu_{0}\right)(F-\widetilde{F})=F^{(1)}+F^{(2)}+F^{(3)}
$$


that belong to $W_{p}^{1}(\Omega)^{*}$ and possess the following properties. The support of $F^{(1)}$ lies in $\Omega_{0}$ and $F^{(1)} \in W_{p^{\prime}}^{-1}\left(\Omega_{0}\right)$. The functional $F^{(2)}$ defined by

$$
W_{p}^{1}(\Omega) \ni v \mapsto\left\langle F^{(2)}, v\right\rangle=\left\langle F,\left(1-\mu_{0}\right) \widetilde{v}\right\rangle,
$$

has support in the set $\left\{x \in \Omega \cap U: z \leq z_{0}\right\}$ and belongs to the space $X_{p}(0,1)^{*}$ in the sense that

$$
\left|\left\langle F^{(2)}, v\right\rangle\right| \leq \text { const } \cdot\|\widetilde{v}\|_{X_{p}(0,1)}
$$

The functional $F^{(3)}$ has support in the set $\left\{x \in \Omega \cap U: z \leq z_{0}\right\}$, we have

$$
\left\langle F^{(3)}, v\right\rangle=\sum_{k \geq 1}\left\langle\mu_{k}(F-\tilde{F}), v\right\rangle, \quad v \in W_{p}^{1}(\Omega)
$$

and the estimate

$$
\left(\sum_{k \geq 1}\left\|\mu_{k}(F-\tilde{F})\right\|_{W_{p^{\prime}}^{-1}\left(\Omega_{k}\right)}^{p^{\prime}}\right)^{1 / p^{\prime}} \leq c\left\|\left(1-\mu_{0}\right)(F-\tilde{F})\right\|_{W_{p}^{1}(\Omega)^{*}}
$$

is true with a positive constant $c$ depending only on $p$ and $\Omega$.

(ii) Suppose that $F_{k} \in W_{p^{\prime}}^{-1}\left(\Omega_{k}\right)$ for $k=1,2, \ldots, \operatorname{supp} F_{k} \subset \Omega_{k},\left\langle F_{k}, v\right\rangle=0$ if $v \in W_{p}^{1}\left(\Omega_{k}\right), v(y, z)$ depends only on $z$, and

$$
\sum_{k \geq 1}\left\|\lambda_{k} F_{k}\right\|_{W_{p^{\prime}}^{p^{\prime}}\left(\Omega_{k}\right)}^{\prime}<\infty
$$

Let $F^{(3)}$ be defined by

$$
W_{p}^{1}(\Omega) \ni v \mapsto\left\langle F^{(3)}, v\right\rangle=\sum_{k \geq 1}\left\langle\lambda_{k} F_{k}, v\right\rangle .
$$

Then $F^{(3)} \in W_{p}^{1}(\Omega)^{*}, \operatorname{supp} F^{(3)} \subset\left\{x \in \Omega \cap U: z \leq z_{0}\right\}$, and the estimate

$$
\left\|F^{(3)}\right\|_{W_{p}^{1}(\Omega)^{*}} \leq c\left(\sum_{k \geq 1}\left\|\lambda_{k} F_{k}\right\|_{W_{p^{\prime}} p^{\prime}\left(\Omega_{k}\right)}^{p^{\prime}}\right)^{1 / p^{\prime}}
$$

is true with a constant $c$ depending only on $p$ and $\Omega$. Furthermore, let $h \in W_{p^{\prime}}^{-1}\left(\Omega_{0}\right)$ and $g \in X_{p}(0,1)^{*}$. Define $F^{(1)}=\mu_{0} h$, and

$$
\left\langle F^{(2)}, v\right\rangle=\left\langle g,\left(1-\mu_{0}\right) \widetilde{v}\right\rangle, \quad v \in W_{p}^{1}(\Omega) .
$$

Then $F^{(1)}, F^{(2)} \in W_{p}^{1}(\Omega)^{*}$, and moreover, $F^{(1)} \in W_{p^{\prime}}^{-1}\left(\Omega_{0}\right)$.

Proof. (i) The fact that $F^{(1)}=\mu_{0} F \in W_{p}^{1}(\Omega)^{*}$ follows from the estimates

$$
\left|\left\langle F^{(1)}, v\right\rangle\right|=\left|\left\langle F, \mu_{0} v\right\rangle\right| \leq c\|F\|_{W_{p}^{1}(\Omega)^{*}}\left\|\mu_{0} v\right\|_{W_{p}^{1}\left(\Omega_{0}\right)} \leq c\|v\|_{W_{p}^{1}\left(\Omega_{0}\right)} .
$$

We consider $F^{(2)}$. Here

$$
\left|\left\langle F^{(2)}, v\right\rangle\right|=\left|\left\langle F,\left(1-\mu_{0}\right) \widetilde{v}\right\rangle\right| \leq c(F)\left\|\left(1-\mu_{0}\right) \widetilde{v}\right\|_{W_{p}^{1}(\Omega \cap U)} \leq c(F)\|\widetilde{v}\|_{X_{p}(0,1)},
$$

so that (2.5) holds true.

We have $F^{(3)} \in W_{p}^{1}(\Omega)^{*}$ because $F^{(1)}, F^{(2)} \in W_{p}^{1}(\Omega)^{*}$. To verify inequality (2.6), for each $k=1,2, \ldots$ we choose an element $v_{k} \in W_{p}^{1}\left(\Omega_{k}\right)$ such that its norm does not exceed 1 and

$$
\left\|\mu_{k}(F-\widetilde{F})\right\|_{W_{p^{\prime}}^{-1}\left(\Omega_{k}\right)} \leq 2\left\langle\mu_{k}(F-\widetilde{F}), v_{k}\right\rangle .
$$


Let $\alpha_{k}=\left\|\mu_{k}(F-\widetilde{F})\right\|_{W_{p^{\prime}}^{-1}\left(\Omega_{k}\right)}$. Clearly, for any positive integer $N$ we obtain

$$
\sum_{k=1}^{N} \alpha_{k}^{p^{\prime}} \leq 2 \sum_{k=1}^{N} \alpha_{k}^{p^{\prime}-1}\left\langle\mu_{k}(F-\widetilde{F}), v_{k}\right\rangle=2\left\langle F-\widetilde{F}, \sum_{k=1}^{N} \alpha_{k}^{p^{\prime}-1} \mu_{k}\left(v_{k}-\stackrel{\circ}{v}_{k}\right)\right\rangle,
$$

where $\stackrel{\circ}{v}_{k}$ is the mean value of $v_{k}$ on $\Omega_{k}$. Observe that $\mu_{k}=\left(1-\mu_{0}\right) \mu_{k}$ for $k \geq 2$. Also, we have $\mu_{1}=\left(1-\mu_{0}\right) \mu_{1}$ on $\left[z_{2}, z_{1}\right]$, and $\mu_{1}=1-\mu_{0}$ on $\left[z_{1}, z_{0}\right]$. Thus, $\mu_{1}$ can be written in the form $\mu_{1}=\left(1-\mu_{0}\right) \nu_{1}$, where

$$
\nu_{1}(z)= \begin{cases}\mu_{1}(z) & \text { if } z \in\left[z_{2}, z_{1}\right] \\ \lambda_{1}(z) & \text { if } z \in\left[z_{1}, z_{0}\right]\end{cases}
$$

We remind the reader that the $\lambda_{k} \in C_{0}^{\infty}\left(\Delta_{k}\right)$ are functions for which $\mu_{k} \lambda_{k}=\mu_{k}, k=$ $1,2, \ldots$ Therefore, $\nu_{1} \in C_{0}^{\infty}\left(z_{2}, z_{0}\right), 0 \leq \nu_{1} \leq 1$. We put $\nu_{k}=\mu_{k}$ for $k=2,3, \ldots$ and rewrite inequality (2.11) in the form

$$
\sum_{k=1}^{N} \alpha_{k}^{p^{\prime}} \leq 2\left\langle\left(1-\mu_{0}\right)(F-\widetilde{F}), \sum_{k=1}^{N} \alpha_{k}^{p^{\prime}-1} \nu_{k}\left(v_{k}-\stackrel{\circ}{v}_{k}\right)\right\rangle .
$$

Assuming that $\nu_{k}(z) v_{k}(x)=0$ outside $\Omega_{k}$, we define

$$
v(x)=\sum_{k=1}^{N} \alpha_{k}^{p^{\prime}-1} \nu_{k}(z) w_{k}(x), \quad x \in \Omega, \quad w_{k}(x)=v_{k}(x)-\stackrel{\circ}{v}_{k},
$$

and estimate the value $\|v\|_{W_{p}^{1}(\Omega)}$. Since every point $x \in \Omega$ belongs to at most two cells $\Omega_{k}, k \geq 1$, it follows that

$$
\|v\|_{W_{p}^{1}(\Omega)}^{p} \leq c \sum_{k=1}^{N} \alpha_{k}^{\left(p^{\prime}-1\right) p}\left\|\nu_{k} w_{k}\right\|_{W_{p}^{1}\left(\Omega_{k}\right)}^{p} .
$$

By using (2.3) and the Poincaré inequality

$$
\left\|w_{k}\right\|_{L_{p}\left(\Omega_{k}\right)} \leq c \varphi\left(z_{k}\right)\left\|\nabla v_{k}\right\|_{L_{p}\left(\Omega_{k}\right)},
$$

we obtain

$$
\left\|\nu_{k} w_{k}\right\|_{W_{p}^{1}\left(\Omega_{k}\right)}^{p} \leq c\left\|\nabla v_{k}\right\|_{L_{p}\left(\Omega_{k}\right)}^{p} .
$$

Since $\left\|\nabla v_{k}\right\|_{L_{p}\left(\Omega_{k}\right)} \leq 1$, estimate (2.14) implies that

$$
\|v\|_{W_{p}^{1}(\Omega)} \leq c\left(\sum_{k=1}^{N} \alpha_{k}^{\left(p^{\prime}-1\right) p}\right)^{1 / p} .
$$

This inequality and (2.13) give the estimate

$$
\sum_{k=1}^{N} \alpha_{k}^{p^{\prime}} \leq c\left\|\left(1-\mu_{0}\right)(F-\widetilde{F})\right\|_{W_{p}^{1}(\Omega)^{*}}\left(\sum_{k=1}^{N} \alpha_{k}^{p^{\prime}}\right)^{1 / p}
$$

with constant independent of $k, \alpha_{k}, N$. Hence, (2.6) follows.

(ii) Let $v \in W_{p}^{1}(\Omega)$. Then

$$
\begin{aligned}
\sum_{k \geq 1}\left|\left\langle\lambda_{k} F_{k}, v\right\rangle\right| & \leq \sum_{k \geq 1}\left|\left\langle\lambda_{k} F_{k}, \sum_{|k-i| \leq 1} \mu_{i}\left(v-\stackrel{\circ}{v}_{k}\right)\right\rangle\right| \\
& \leq \sum_{k \geq 1} \sum_{|k-i| \leq 1}\left\|\lambda_{k} F_{k}\right\|_{W_{p^{\prime}}^{-1}\left(\Omega_{k}\right)}\left\|\mu_{i}\left(v-\stackrel{\circ}{v}_{k}\right)\right\|_{W_{p}^{1}\left(\Omega_{k}\right)},
\end{aligned}
$$


where $\stackrel{\circ}{v}_{k}$ is the mean value of $v$ on $\Omega_{k}$. Since $|k-i| \leq 1$, we have

$$
\left\|\mu_{i}\left(v-\stackrel{\circ}{v}_{k}\right)\right\|_{W_{p}^{1}\left(\Omega_{k}\right)} \leq c\|\nabla v\|_{L_{p}\left(\Omega_{k}\right)}
$$

whence

$$
\sum_{k \geq 1}\left|\left\langle\lambda_{k} F_{k}, v\right\rangle\right| \leq c\left(\sum_{k \geq 1}\left\|\lambda_{k} F_{k}\right\|_{W_{p^{\prime}}^{-1}\left(\Omega_{k}\right)}^{p^{\prime}}\right)^{1 / p^{\prime}}\left(\sum_{k \geq 1}\|\nabla v\|_{L_{p}\left(\Omega_{k}\right)}^{p}\right)^{1 / p} .
$$

Thus, the functional (2.8) is well defined under the assumption (2.7), and inequality (2.9) is valid.

Let $h \in W_{p^{\prime}}^{-1}\left(\Omega_{0}\right)$. Then

$$
\left|\left\langle F^{(1)}, v\right\rangle\right|=\left|\left\langle h, \mu_{0} v\right\rangle\right| \leq c(h)\left\|\mu_{0} v\right\|_{W_{p}^{1}\left(\Omega_{0}\right)} \leq c(h)\|v\|_{W_{p}^{1}\left(\Omega_{0}\right)},
$$

so that $F^{(1)} \in W_{p^{\prime}}^{-1}\left(\Omega_{0}\right)$.

Finally, if $g \in X_{p}(0,1)^{*}$, then

$$
\begin{aligned}
\left|\left\langle F^{(2)}, v\right\rangle\right| & =\left|\left\langle g,\left(1-\mu_{0}\right) \widetilde{v}\right\rangle\right| \leq\|g\|_{X_{p}(0,1) *}\left\|\left(1-\mu_{0}\right) \widetilde{v}\right\|_{X_{p}(0,1)} \\
& \leq c(g)\left(\int_{0}^{1}\left(|\widetilde{v}(z)|^{p}+\left|\widetilde{v}^{\prime}(z)\right|^{p}\right) \varphi(z)^{n-1} d z\right)^{1 / p} .
\end{aligned}
$$

The right-hand side of this inequality is not greater than $c(g)\|v\|_{W_{p}^{1}(\Omega \cap U)}$ by Lemma 2 . Consequently, $F^{(2)} \in W_{p}^{1}(\Omega)^{*}$. The proof of the theorem is complete.

Now we state some corollaries to the theorem proved above. By unifying the content of $\S 1$ with Theorem 1 , we arrive at the following assertion.

Corollary 1. Let $\Omega$ be a domain with an outward peak. If problem (1.5) is solvable, then $F$ can be extended uniquely up to a functional on $W_{p}^{1}(\Omega)$ that is representable as the sum of three summands $F^{(1)}, F^{(2)}, F^{(3)}$ described in statement (i) of the theorem. Conversely, if $F$ is a functional on $W_{p}^{1}(\Omega)$ equal to the sum of three terms $F^{(1)}, F^{(2)}$, $F^{(3)}$ as in statement (ii) of the theorem, then problem (1.5) is uniquely solvable.

We say that positive quantities $a, b$ are equivalent (and write $a \sim b$ ) if $c_{1} \leq a / b \leq c_{2}$ for some positive constants $c_{1}, c_{2}$ independent of $a$ and $b$.

Corollary 2. Let $F$ be a linear functional defined on $W_{p}^{1}(\Omega)$, and let $\widetilde{F}$ be defined on the set of functions with support in $\Omega \cap U$ by

$$
\langle\widetilde{F}, v\rangle=\langle F, \widetilde{v}\rangle .
$$

Then $\left(1-\mu_{0}\right)(F-\widetilde{F})$ is a continuous functional on $W_{p}^{1}(\Omega)$ if and only if

$$
\sum_{k \geq 1}\left\|\mu_{k}(F-\widetilde{F})\right\|_{W_{p^{\prime}}^{-1}\left(\Omega_{k}\right)}^{p^{\prime}}<\infty
$$

Furthermore, the following equivalence relation,

$$
\left(\sum_{k \geq 1}\left\|\mu_{k}(F-\widetilde{F})\right\|_{W_{p^{\prime}}^{-1}\left(\Omega_{k}\right)}^{p^{\prime}}\right)^{1 / p^{\prime}} \sim\left\|\left(1-\mu_{0}\right)(F-\widetilde{F})\right\|_{W_{p}^{1}(\Omega)^{*}},
$$

holds true with constants depending only on $p$ and $\Omega$. 
Proof. A lower bound for $\left\|\left(1-\mu_{0}\right)(F-\widetilde{F})\right\|_{W_{p}^{1}(\Omega)^{*}}$ was obtained in statement (i) of the theorem. We deduce the upper one. Consider functionals $F_{k}=\mu_{k}(F-\widetilde{F}), k=1,2, \ldots$, that satisfy (2.15). It is clear that $\operatorname{supp} F_{k} \subset \Omega_{k}$ and that $F_{k}$ vanishes on all functions in $W_{p}^{1}\left(\Omega_{k}\right)$ depending only on the variable $z$. Since $\lambda_{k} \mu_{k}=\mu_{k}$, it follows that $\lambda_{k} F_{k}=$ $\mu_{k}(F-\widetilde{F})=F_{k}$. Therefore, statement (ii) of Theorem 1 gives

$$
\left\|\sum_{k \geq 1} F_{k}\right\|_{W_{p}^{1}(\Omega)^{*}} \leq c\left(\sum_{k \geq 1}\left\|F_{k}\right\|_{W_{p^{\prime}}^{-1}\left(\Omega_{k}\right)}^{p^{\prime}}\right)^{1 / p^{\prime}},
$$

thus completing the proof.

The preceding assertion enables us to prove that $F-\widetilde{F} \in W_{p}^{1}(\Omega)^{*}$ if $F \in L_{q^{\prime}}(\Omega)$ with the minimal possible exponent $q^{\prime}$, and also to establish the continuity of the linear map

$$
W_{p}^{1}(\Omega) \ni v \mapsto v-\widetilde{v} \in L_{q}(\Omega \cap U)
$$

with the maximal Sobolev exponent $q$.

Corollary 3. Let $q=n p /(n-p)$ for $p<n, q \in[p, \infty)$ for $p=n$, and $q=\infty$ for $p>n$. Suppose also that $q^{-1}+q^{\prime-1}=1$. If $F \in L_{q^{\prime}}(\Omega)$, then the functional defined by

$$
W_{p}^{1}(\Omega) \ni v \mapsto\left\langle\left(1-\mu_{0}\right)(F-\widetilde{F}), v\right\rangle=\int_{\Omega}\left(1-\mu_{0}(x)\right) F(x)(v(x)-\widetilde{v}(z)) d x
$$

belongs to $W_{p}^{1}(\Omega)^{*}$, and

$$
\left\|\left(1-\mu_{0}\right)(F-\widetilde{F})\right\|_{W_{p}^{1}(\Omega)^{*}} \leq c\|F\|_{L_{q^{\prime}}(\Omega)}
$$

with a constant independent of $F$. Furthermore, for all $v \in W_{p}^{1}(\Omega \cap U)$ the estimate

$$
\|v-\widetilde{v}\|_{L_{q}(\Omega \cap U)} \leq c\|v\|_{W_{p}^{1}(\Omega \cap U)}
$$

is true with constant independent of $v$.

Proof. Corollary 2 shows that we should bound the sum on the left in (2.15). If $v \in$ $W_{p}^{1}\left(\Omega_{k}\right), k \geq 1$, then, by the Hölder inequality,

$$
\left|\left\langle\mu_{k}(F-\widetilde{F}), v\right\rangle\right|=\left|\int_{\Omega} F(x) \mu_{k}(z)(v(x)-\widetilde{v}(z)) d x\right| \leq\|F\|_{L_{q^{\prime}}\left(\Omega_{k}\right)}\|v-\widetilde{v}\|_{L_{q}\left(\Omega_{k}\right)} .
$$

Since $\Omega_{k}$ is a domain of class $C^{0,1}$, the Sobolev theorem [3, §8] applies, so that for any $u \in W_{p}^{1}\left(\Omega_{k}\right)$ we have

$$
\|u\|_{L_{q}\left(\Omega_{k}\right)} \leq c \varphi\left(z_{k}\right)^{\frac{n}{q}-\frac{n}{p}}\|u\|_{L_{p}\left(\Omega_{k}\right)}+c \varphi\left(z_{k}\right)^{1+\frac{n}{q}-\frac{n}{p}}\|\nabla u\|_{L_{p^{\prime}}\left(\Omega_{k}\right)} .
$$

First we consider the case where $p \in(1, n)$. Then, by taking $u=v-\widetilde{v}$ in the preceding inequality, we obtain

$$
\|v-\widetilde{v}\|_{L_{q}\left(\Omega_{k}\right)} \leq c \varphi\left(z_{k}\right)^{-1}\|v-\widetilde{v}\|_{L_{p}\left(\Omega_{k}\right)}+c\|\nabla(v-\widetilde{v})\|_{L_{p}\left(\Omega_{k}\right)} .
$$

By the Poincaré inequality, for every section of $\Omega_{k}$ by a plane $z=$ const, we obtain

$$
\begin{aligned}
\|v-\widetilde{v}\|_{L_{p}\left(\Omega_{k}\right)} & =\left(\int_{z_{k+1}}^{z_{k-1}} d z \int_{y / \varphi(z) \in \omega}|v(y, z)-\widetilde{v}(z)|^{p} d y\right)^{1 / p} \\
& \leq c\left(\int_{z_{k+1}}^{z_{k-1}} \varphi(z)^{p} d z \int_{y / \varphi(z) \in \omega}\left|\nabla_{y} v(y, z)\right|^{p} d y\right)^{1 / p} .
\end{aligned}
$$


Hence, the first term on the right in (2.19) does not exceed $c\|\nabla v\|_{L_{p}\left(\Omega_{k}\right)}$. By Lemma 2, the last term in (2.19) admits the same majorant. Thus, for $p<n$ we have

$$
\|v-\widetilde{v}\|_{L_{q}\left(\Omega_{k}\right)} \leq c\|\nabla v\|_{L_{p}\left(\Omega_{k}\right)}, \quad k \geq 1 .
$$

A similar argument for $p \geq n$ gives

$$
\|v-\widetilde{v}\|_{L_{q}\left(\Omega_{k}\right)} \leq c \varphi\left(z_{k}\right)^{\max \left\{\frac{n}{q}, 1-\frac{n}{p}\right\}}\|\nabla v\|_{L_{p}\left(\Omega_{k}\right)} .
$$

Therefore, inequality (2.20) holds true also for $p \geq n$. Unifying (2.18) and (2.20), we arrive at

$$
\left\|\mu_{k}(F-\widetilde{F})\right\|_{W_{p^{\prime}}^{-1}\left(\Omega_{k}\right)} \leq c\|F\|_{L_{q^{\prime}}\left(\Omega_{k}\right)} .
$$

Now Corollary 2 implies that

$$
\left\|\left(1-\mu_{0}\right)(F-\widetilde{F})\right\|_{W_{p}^{1}(\Omega)^{*}} \leq c\left(\sum_{k \geq 1}\|F\|_{L_{q^{\prime}}\left(\Omega_{k}\right)}^{p^{\prime}}\right)^{1 / p^{\prime}} .
$$

By using the algebraic inequality

$$
\left(\sum_{k \geq 1} a_{k}^{\gamma}\right)^{1 / \gamma} \leq\left(\sum_{k \geq 1} a_{k}^{\beta}\right)^{1 / \beta}, \quad a_{k} \geq 0, \gamma>\beta>0,
$$

and keeping in mind that $q^{\prime} \leq p^{\prime}$, we dominate the right-hand side of (2.21) by the expression

$$
c\left(\sum_{k \geq 1}\|F\|_{L_{q^{\prime}}\left(\Omega_{k}\right)}^{q^{\prime}}\right)^{1 / q^{\prime}} \leq c\|F\|_{L_{q^{\prime}}(\Omega)},
$$

and inequality (2.16) follows.

Now we turn to (2.17). With the help of (2.22), from (2.20) we obtain

$$
\begin{aligned}
\left(\int_{\Omega^{\prime}}|v(x)-\widetilde{v}(z)|^{q} d x\right)^{1 / q} & \leq\left(\sum_{k \geq 1} \int_{\Omega_{k}}|v-\widetilde{v}|^{q} d x\right)^{1 / q} \\
& \leq c\left(\sum_{k \geq 1}\|\nabla v\|_{L_{p}\left(\Omega_{k}\right)}^{p}\right)^{1 / p} \leq c\|\nabla v\|_{L_{p}(\Omega \cap U)},
\end{aligned}
$$

where $\Omega^{\prime}=\bigcup_{k \geq 1} \Omega_{k}$. It remains to observe that the estimate

$$
\left(\int_{(\Omega \cap U) \backslash \bar{\Omega}^{\prime}}|v(x)-\widetilde{v}(z)|^{q} d x\right)^{1 / q} \leq c\|\nabla v\|_{L_{p}(\Omega \cap U)}
$$

can be deduced from the Sobolev theorem for the domain $(\Omega \cap U) \backslash \bar{\Omega}^{\prime}$ of class $C^{0,1}$ and the Poincaré inequality for the section of $\Omega \cap U$ by the plane $z=$ const. The proof of the corollary is complete.

The assertion below is a consequence of Theorem 1 and Corollary 3 .

Proposition 1. Let $p \leq q \leq p n /(n-p)$ for $p<n, q \in[p, \infty)$ for $p=n$, and $p \leq q \leq \infty$ for $p>n$, and let $q^{-1}+q^{-1}=1$. Suppose that $\Omega \subset \mathbb{R}^{n}$ is a domain with an outward peak. The following statements are equivalent.

(A) The Neumann problem (1.5) is solvable for all $F \in L_{q^{\prime}}(\Omega)$.

(B) The space $W_{p}^{1}(\Omega)$ is imbedded continuously in $L_{q}(\Omega)$.

(C) For all $F \in L_{q^{\prime}}(\Omega)$, the map

$$
W_{p}^{1}(\Omega) \ni v \mapsto \int_{\Omega} F v d x
$$

is a continuous linear functional. 
(D) The map $W_{p}^{1}(\Omega \cap U) \ni v \mapsto \widetilde{v} \in L_{q}(\Omega)$ is continuous.

(E) If $u \in X_{p}(0,1)$ and $u(1)=0$, then

$$
\left(\int_{0}^{1}|u(z)|^{q} \varphi(z)^{n-1} d z\right)^{1 / q} \leq \mathrm{const} \cdot\left(\int_{0}^{1}\left|u^{\prime}(z)\right|^{p} \varphi(z)^{n-1} d z\right)^{1 / p} .
$$

Proof. (A) $\Rightarrow$ (B). We put

$$
V=\left\{v \in W_{p}^{1}(\Omega) \cap L_{\infty}(\Omega) \cap C^{\infty}(\Omega):\|v\|_{W_{p}^{1}(\Omega)} \leq 1\right\}
$$

and, for each $v \in V$, consider a functional

$$
L_{q^{\prime}}(\Omega) \ni F \mapsto \Phi_{v}(F)=\int_{\Omega} F v d x .
$$

Clearly, $\Phi_{v}$ is continuous, and (1.5) implies that $\left\{\Phi_{v}(F)\right\}$ is a bounded set for any fixed $F \in L_{q^{\prime}}(\Omega)$. Hence, $\left\|\Phi_{v}\right\|=\|v\|_{L_{q}(\Omega)} \leq$ const for $v \in V$, and we have

$$
\|v\|_{L_{q}(\Omega)} \leq \mathrm{const} \cdot\|v\|_{W_{p}^{1}(\Omega)}
$$

for all $v \in W_{p}^{1}(\Omega) \cap L_{\infty}(\Omega) \cap C^{\infty}(\Omega)$. Since this set is dense in $W_{p}^{1}(\Omega)$, the preceding inequality is true for all $v \in W_{p}^{1}(\Omega)$.

$(\mathrm{B}) \Rightarrow(\mathrm{C})$. The Hölder inequality yields

$$
\left|\int_{\Omega} F v d x\right| \leq\|F\|_{L_{q^{\prime}}(\Omega)}\|v\|_{L_{q}(\Omega)} \leq c\|F\|_{L_{q^{\prime}}(\Omega)}\|v\|_{W_{p}^{1}(\Omega)},
$$

whence the functional $W_{p}^{1}(\Omega) \ni v \mapsto \int_{\Omega} F v d x$ is continuous. in $\S 1$.

$(\mathrm{C}) \Rightarrow(\mathrm{A})$. The solvability of problem (1.5) under assumption (C) was established

The equivalence of statements (B) and (D) follows from Corollary 3.

$(\mathrm{D}) \Rightarrow(\mathrm{E})$. Let $u \in X_{p}(0,1), u(1)=0$; we define $v(x)=u(z)$ for $x \in \Omega \cap U$. Then (D) implies

$$
\left(\int_{0}^{1}|u(z)|^{q} \varphi(z)^{n-1} d z\right)^{1 / q} \leq c\left(\int_{0}^{1}\left(\left|u^{\prime}(z)\right|^{p}+|u(z)|^{p}\right) \varphi(z)^{n-1} d z\right)^{1 / p} .
$$

Next we note that the "Friedrichs inequality"

$$
\int_{0}^{1}|u(z)|^{p} \varphi(z)^{n-1} d z \leq \int_{0}^{1}\left|u^{\prime}(z)\right|^{p} \varphi(z)^{n-1} d z
$$

is a simple consequence of the Hölder inequality and the monotonicity of $\varphi$. Thus, (2.23) follows from (2.24).

$(\mathrm{E}) \Rightarrow(\mathrm{D})$. Let $\lambda$ be a smooth function on $(0,1)$ such that $0 \leq \lambda \leq 1, \lambda(z)=1$ for $z \in(0,1 / 2]$, and $\lambda(z)=0$ for $z \in[2 / 3,1)$. Let $v \in W_{p}^{1}(\Omega \cap U)$, and let $u(z)=\widetilde{v}(z)$. Then, by (2.23) and Lemma 2 ,

$$
\left(\int_{0}^{1}|\lambda(z) \widetilde{v}(z)|^{q} \varphi(z)^{n-1} d z\right)^{1 / q} \leq c\left(\int_{0}^{1}\left|\frac{d}{d z}(\lambda \widetilde{v})\right|^{p} d z\right)^{1 / p} \leq c\|v\|_{W_{p}^{1}(\Omega \cap U)} .
$$

Furthermore, from the embedding $W_{p}^{1}(1 / 2,1) \subset L_{q}(1 / 2,1)$ and the same lemma we obtain

$$
\begin{aligned}
& \left(\int_{0}^{1}|(1-\lambda(z)) \widetilde{v}(z)|^{q} \varphi(z)^{n-1} d z\right)^{1 / q} \leq c\left(\int_{1 / 2}^{1}|\widetilde{v}(z)|^{q} d z\right)^{1 / q} \\
& \quad \leq c\|\widetilde{v}\|_{W_{p}^{1}(1 / 2,1)} \leq c\left(\int_{1 / 2}^{1}\left(|\widetilde{v}(z)|^{p}+\left|\widetilde{v}^{\prime}(z)\right|^{p}\right) \varphi(z)^{n-1} d z\right)^{1 / p} \leq c\|v\|_{W_{p}^{1}(\Omega \cap U)} .
\end{aligned}
$$


Thus,

$$
\|\widetilde{v}\|_{L_{q}(\Omega \cap U)} \leq c\|v\|_{W_{p}^{1}(\Omega \cap U)},
$$

which completes the proof of Proposition 1.

Remark 1. By using a well-known condition for the validity of Hardy's weighted inequality (2.23) (see, e.g., [5, 1.3]), we supplement Proposition 1 in the following way. Each of the statements $(\mathrm{A})-(\mathrm{E})$ above is equivalent to the inequality 2

$$
\sup _{r \in(0,1)}\left(\int_{0}^{r} \varphi(z)^{n-1} d z\right)^{1 / q}\left(\int_{r}^{1} \varphi(z)^{\frac{1-n}{p-1}} d z\right)^{(p-1) / p}<\infty .
$$

For $q=\infty$, this inequality should be replaced by

$$
\int_{0}^{1} \varphi(z)^{\frac{1-n}{p-1}} d z<\infty
$$

\section{§3. Auxiliary assertions}

In this section, we state some lemmas to be used in the proof of Theorem 2 in $\S 4$. Preliminarily, we introduce some notation.

Let $\Omega$ be a domain with an outward peak in the sense of the definition at the beginning of $\S 2$.

For simplicity of presentation, in the present and the next section we assume that $\bar{\omega} \subset B_{1}$, and that $\varphi^{\prime}(z) \leq 1 / 2$ for almost all $z \in(0,1)$. Furthermore, assuming that $(2.1)$ is fulfilled, we put

$$
\Gamma=\{x=(y, z): z \in(0,1), y / \varphi(z) \in \partial \omega\} .
$$

If $f \in T W_{p}^{1}(\Omega)^{*}$ and $\lambda$ is a Lipschitz function on $\partial \Omega$, we define

$$
\langle\lambda f, v\rangle=\langle f, \lambda v\rangle, \quad v \in T W_{p}^{1}(\Omega) .
$$

Let $v$ be a function defined on $\Gamma$. Then its mean value on the section of $\Gamma$ by the hyperplane $z=$ const is defined as

$$
\bar{v}(z)=\frac{1}{|\gamma|} \int_{\gamma} v(\varphi(z) y, z) d \gamma(y), \quad \gamma=\partial \omega
$$

where $|\gamma|$ is the $(n-2)$-dimensional area of $\gamma$.

If $f \in T W_{p}^{1}(\Omega)^{*}$ and the support of $v \in T W_{p}^{1}(\Omega)$ lies in $\Gamma$, then, by definition, $\langle\bar{f}, v\rangle=\langle f, \bar{v}\rangle$.

Let $\left\{\mu_{k}\right\}_{k>0}$ be the partition of unity for the interval $(0,1]$ constructed in $\S 2$. We shall use it below, but, along with condition (2.3), we shall require that the number $z_{0}$ be so small that

$$
\varphi(z-2 \varphi(z))>3 \varphi(z) / 4 \text { and } \varphi(z)<z / 4
$$

for $z<2 z_{0}$.

We introduce the surfaces

$$
\Gamma_{k}=\left\{(y, z) \in \Gamma: z \in \Delta_{k}\right\}, \quad \Delta_{k}=\left(z_{k+1}, z_{k-1}\right), \quad k=1,2, \ldots
$$

and

$$
\Gamma_{0}=\partial \Omega \backslash\left\{x \in \bar{\Gamma}: z \leq z_{1}\right\} .
$$

We observe that the above partition of unity for the interval $(0,1]$ induces a partition of unity for $\partial \Omega \backslash\{O\}$ : we put $\mu_{0}=1$ on $\Gamma_{0} \backslash \Gamma, \mu_{k}(x)=\mu_{k}(z)$ for $x \in \Gamma_{k} \cap \Gamma, k \geq 0$, and $\mu_{k}=0$ on $\partial \Omega \backslash \Gamma, k \geq 1$. This partition of unity is subordinate to the covering $\left\{\Gamma_{k}\right\}_{k \geq 0}$

\footnotetext{
${ }^{2}$ However, the equivalence of the continuity of the imbedding $W_{p}^{1}(\Omega) \rightarrow L_{q}(\Omega)$ to the same inequality is also well known, see [5 4.8.5], and was obtained with the help of capacitary "isoperimetric" inequalities.
} 
in the sense that $\operatorname{dist}\left(\operatorname{supp} \mu_{k}, \partial \Omega \backslash \Gamma_{k}\right)>0, k \geq 0$. We also introduce a set of functions $\left\{\lambda_{k}\right\}_{k \geq 1}$ such that $\lambda_{k} \in C_{0}^{\infty}\left(\Delta_{k}\right)$ and $\lambda_{k} \mu_{k}=\mu_{k}$ for all $k \geq 1$.

We say that a functional $f \in T W_{p}^{1}(\Omega)^{*}$ has support in $\Gamma_{k}$ (and write $\operatorname{supp} f \subset \Gamma_{k}$ ) if $\left.v\right|_{\Gamma_{k}}=0$ implies $\langle f, v\rangle=0$.

For a domain with outward peak, the space $T W_{p}^{1}(\Omega)$ admits an explicit description (see [7, 4, and [8, 7.2]); namely, this space consists of all functions on $\partial \Omega$ with finite norm

$$
\left(\|v\|_{W_{p}^{1-1 / p}\left(\Gamma_{0}\right)}^{p}+\int_{\Gamma}|v(x)|^{p} \varphi(z) d s_{x}+|v|_{p, \Gamma}^{p}\right)^{1 / p}
$$

where

$$
|v|_{p, \Gamma}=\left(\iint_{\Gamma \times \Gamma}|v(x)-v(\xi)|^{p} \chi\left(\frac{|z-\zeta|}{M(z, \zeta)}\right) \frac{d s_{x} d s_{\xi}}{|x-\xi|^{n+p-2}}\right)^{1 / p},
$$

$x=(y, z), \xi=(\eta, \zeta), M(z, \zeta)=\max \{\varphi(z), \varphi(\zeta)\}, \chi$ is the characteristic function of the interval $(0,1)$, and $d s_{x}, d s_{\xi}$ are the area elements on $\Gamma$. Moreover, the norm (3.3) is equivalent to the norm in $T W_{p}^{1}(\Omega)$. This equivalence of norms remains valid if the surface $\Gamma_{0}$ in (3.3) is replaced by the surface $\partial \Omega \backslash\{x \in \bar{\Gamma}: z \leq \delta\}, \delta \in(0,1)$, and the integral over $\Gamma$ is dropped (see 7 ).

We need some auxiliary assertions to prove Theorem 2 in the next section.

Lemma 3. For $v \in L_{p, \text { loc }}(0,1)$, the equivalence relation

$$
\begin{aligned}
& \iint_{\Gamma \times \Gamma}|v(z)-v(\zeta)|^{p} \chi\left(\frac{|z-\zeta|}{M(z, \zeta)}\right) \frac{d s_{x} d s_{\xi}}{|x-\xi|^{n+p-2}} \\
& \quad \sim \iint_{\{z, \zeta \in(0,1)\}}|v(z)-v(\zeta)|^{p} \frac{M(z, \zeta)^{n-2}}{|z-\zeta|^{p}} \chi\left(\frac{|z-\zeta|}{M(z, \zeta)}\right) d z d \zeta
\end{aligned}
$$

holds true with constants independent of $v$, and if $v \in T W_{p}^{1}(\Omega)$, then

$$
|\bar{v}|_{p, \Gamma} \leq c(p, \Omega)\|v\|_{T W_{p}^{1}(\Omega)} .
$$

Proof. The left-hand side of (3.5) is equivalent to

$$
\int_{0}^{1} d z \int_{z-\varphi(z)}^{z}|v(z)-v(\zeta)|^{p}(\varphi(z) \varphi(\zeta))^{n-2} d \zeta \iint_{\gamma \times \gamma} \frac{d \gamma(y) d \gamma(\eta)}{(|z-\zeta|+|\varphi(z) y-\varphi(\zeta) \eta|)^{n+p-2}}
$$

and for $y, \eta \in \gamma$ we have

$$
|z-\zeta|+|\varphi(z) y-\varphi(\zeta) \eta| \sim|z-\zeta|+\varphi(\zeta)|y-\eta| .
$$

Hence,

$$
\begin{aligned}
(\varphi(z) \varphi(\zeta))^{n-2} \iint_{\gamma \times \gamma} & \frac{d \gamma(y) d \gamma(\eta)}{(|z-\zeta|+|\varphi(z) y-\varphi(\zeta) \eta|)^{n+p-2}} \\
& \sim \frac{(\varphi(z) \varphi(\zeta))^{n-2}}{|z-\zeta|^{n+p-2}} \int_{\gamma} d \gamma(y) \int_{\gamma} \frac{d \gamma(\eta)}{(1+\lambda|y-\eta|)^{n+p-2}}
\end{aligned}
$$

where $\lambda=\varphi(\zeta)|z-\zeta|^{-1}$. For fixed $y \in \gamma$, we make the change of variables $\eta=y+\lambda^{-1} t$ in the last integral over $\gamma$. Then the expression on the right in (3.7) takes the form

$$
\frac{\varphi(z)^{n-2}}{|z-\zeta|^{p}} \int_{\gamma} d \gamma(y) \int_{S_{\lambda}} \frac{d S_{\lambda}(t)}{(1+|t|)^{n+p-2}} .
$$

Here $S_{\lambda}$ is the surface $\{t: t / \lambda+y \in \gamma\}$, and $d S_{\lambda}(t)$ is the element of $(n-2)$-dimensional area. It remains to note that $\varphi(z) \sim \varphi(\zeta)$ for $|z-\zeta|<M(z, \zeta)$, whence $\lambda \geq$ const $>0$, 
and the last integral is bounded from above and from below uniformly in $\lambda$. So, (3.5) follows.

We turn to (3.6). By Hölder's inequality and (3.1), we have

$$
|\bar{v}(z)-\bar{v}(\zeta)|^{p} \leq c \int_{\gamma}|v(\varphi(z) y, z)-v(\varphi(\zeta) y, \zeta)|^{p} d \gamma(y) .
$$

Thus, in view of (3.5), inequality (3.6) is a consequence of the inequality

$$
I_{\gamma}(v) \leq c\|v\|_{T W_{p}^{1}(\Omega)}
$$

where

$$
I_{\gamma}(v)^{p}=\int_{0}^{1} \varphi(z)^{n-2} d z \int_{z-\varphi(z)}^{z} \frac{d \zeta}{|z-\zeta|^{p}} \int_{\gamma}|v(\varphi(z) y, z)-v(\varphi(\zeta) y, \zeta)|^{p} d \gamma(y) .
$$

Suppose $v=\left.u\right|_{\partial \Omega}$ for some $u \in W_{p}^{1}(\Omega)$; we check that

$$
I_{\gamma}(v) \leq c\|\nabla u\|_{L_{p}(\Omega \cap U)} .
$$

This estimate is known if $\omega=\{y:|y|<1\}$ (see [8, Lemma 7.5/3, inequality (7.5/16)]). If the domain $\omega$ is starlike with respect to a ball centered at the origin, the proof of (3.9) repeats the argument for the case of $\omega=\{y:|y|<1\}$ almost word for word. We omit it. Suppose that $\omega$ is starlike with respect to a ball centered at $y_{0} \in \omega, y_{0} \neq 0$. The transformation

$$
x=(y, z) \mapsto x^{\prime}=\left(y^{\prime}, z^{\prime}\right): z^{\prime}=z, y^{\prime}=y-\varphi(z) y_{0},
$$

converts the domain $\Omega \cap U$ to

$$
\Omega^{\prime}=\left\{\left(y^{\prime}, z^{\prime}\right): z^{\prime} \in(0,1), y^{\prime} / \varphi\left(z^{\prime}\right) \in \omega-y_{0}\right\},
$$

where $\omega-y_{0}$ is starlike with respect to a ball centered at the origin. Given $u \in W_{p}^{1}(\Omega)$, we define $\widetilde{u}$ on $\Omega^{\prime}$ by

$$
\Omega^{\prime} \ni\left(y^{\prime}, z^{\prime}\right) \mapsto \widetilde{u}\left(y^{\prime}, z^{\prime}\right)=u\left(y^{\prime}+\varphi\left(z^{\prime}\right) y_{0}, z^{\prime}\right) .
$$

Then $\widetilde{u} \in W_{p}^{1}\left(\Omega^{\prime}\right)$, and since $\left|\nabla_{x^{\prime}} \widetilde{u}\right| \sim\left|\nabla_{x} u\right|$ and $d x^{\prime}=d x$, it follows that

$$
\|\nabla \widetilde{u}\|_{L_{p}\left(\Omega^{\prime}\right)} \sim\|\nabla u\|_{L_{p}(\Omega)} \text {. }
$$

We also have

$$
I_{\gamma}(v)=I_{\gamma-y_{0}}(\widetilde{v}), \quad \widetilde{v}=\left.\widetilde{u}\right|_{\partial \Omega^{\prime}}
$$

The above observations show that

$$
I_{\gamma}(v)=I_{\gamma-y_{0}}(\widetilde{v}) \leq c\|\nabla \widetilde{u}\|_{L_{p}\left(\Omega^{\prime}\right)} \leq c\|\nabla u\|_{L_{p}(\Omega \cap U)} .
$$

Finally, let $\omega$ be the union of a finite number of domains starlike with respect to a ball: $\omega=\bigcup_{i=1}^{N} \omega_{i}$. In this case, $\gamma=\partial \omega \subset \bigcup_{i=1}^{N} \gamma_{i}, \gamma_{i}=\partial \omega_{i}$, whence

$$
I_{\gamma}(v)^{p} \leq \sum_{i=1}^{N} I_{\gamma_{i}}(v)^{p} \leq c\|\nabla u\|_{L_{p}(\Omega \cap U)}^{p} .
$$

It remains to note that any domain $\omega \in C^{0,1}$ can be represented as the union of a finite number of domains starlike with respect to a ball [6]. So, estimate (3.9) is proved. To obtain (3.8), we observe that any function $v$ with finite norm $\|v\|_{T W_{p}^{1}(\Omega)}$ can be extended inside $\Omega$ so that this extension $u$ is subject to

$$
\|u\|_{W_{p}^{1}(\Omega)} \leq c\|v\|_{T W_{p}^{1}(\Omega)} .
$$

Then (3.8) is a consequence of (3.9). This concludes the proof. 
Lemma 4. If $v \in T W_{p}^{1}(\Omega), v(x)=0$ for $x \in \Gamma, z>z_{0}$, then the seminorm $|v|_{p, \Gamma}$ is equivalent to

$$
\left(\iint_{\Gamma \times \Gamma}|v(x)-v(\xi)|^{p} \chi\left(\frac{|z-\zeta|}{2 M(z, \zeta)}\right) \frac{d s_{x} d s_{\xi}}{|x-\xi|^{n+p-2}}\right)^{1 / p}
$$

(we use the same notation as in (3.4)).

Proof. It suffices to prove the estimate

$$
\iint_{S}|v(x)-v(\xi)|^{p} \frac{d s_{x} d s_{\xi}}{|x-\xi|^{n+p-2}} \leq c|v|_{p, \Gamma}^{p},
$$

where

$$
S=\{(x, \xi) \in \Gamma \times \Gamma: \varphi(z)<z-\zeta<2 \varphi(z)\} .
$$

Since $v(x)=0$ for $z>z_{0}$, the integrand in (3.10) is nonzero only if $\psi(z)<z_{0}$, where $\psi(z)=z-2 \varphi(z)$. By (3.2), we have $\psi\left(2 z_{0}\right) \geq z_{0}$, and the monotonicity of $\psi$ shows that the inequality $\psi(z)<z_{0}$ can be fulfilled only for $z<2 z_{0}$. Thus, the integrand in (3.10) does not vanish only if $z \in\left(0,2 z_{0}\right)$. We also observe that $|x-\xi| \sim \varphi(z)$ for $(x, \xi) \in S$. We shall prove (3.10) by what is called fictitious integration. Let $x^{\prime}$ and $\xi^{\prime}$ be defined by

$$
\begin{aligned}
& x^{\prime}=\left(y^{\prime}, z^{\prime}\right) \in \Gamma: z^{\prime} \in \delta_{1}(z)=(z-\varphi(z), z-3 \varphi(z) / 4), \\
& \xi^{\prime}=\left(\eta^{\prime}, \zeta^{\prime}\right) \in \Gamma: \zeta^{\prime} \in \delta_{2}(z)=(z-3 \varphi(z) / 2, z-5 \varphi(z) / 4) .
\end{aligned}
$$

Then

$$
0<z-z^{\prime}<\varphi(z)=M(z, \zeta)
$$

By (3.2), we also have

$$
0<z^{\prime}-\zeta^{\prime}<3 \varphi(z) / 4<\varphi(z-3 \varphi(z) / 2)<\varphi\left(z^{\prime}\right)=M\left(z^{\prime}, \zeta^{\prime}\right)
$$

and

$$
\left|\zeta^{\prime}-\zeta\right| \leq 3 \varphi(z) / 4<\varphi\left(\zeta^{\prime}\right) \leq M\left(\zeta, \zeta^{\prime}\right)
$$

Integrating the inequality

$$
c|v(x)-v(\xi)|^{p} \leq\left|v(x)-v\left(x^{\prime}\right)\right|^{p}+\left|v\left(x^{\prime}\right)-v\left(\xi^{\prime}\right)\right|^{p}+\left|v\left(\xi^{\prime}\right)-v(\xi)\right|^{p}
$$

with respect to $x^{\prime}, \xi^{\prime}$ and using the fact that each quantity

$$
\left|x-x^{\prime}\right|,\left|x^{\prime}-\xi^{\prime}\right|,\left|\xi^{\prime}-\xi\right|
$$

is less than or equal to $c|x-\xi|$ and $|x-\xi| \sim \varphi(z)$, we obtain

$$
\begin{aligned}
c \frac{|v(x)-v(\xi)|^{p}}{|x-\xi|^{n+p-2}} \leq & \frac{1}{\varphi(z)^{n-1}} \int_{\left\{x^{\prime} \in \Gamma: z^{\prime} \in \delta_{1}(z)\right\}}\left|v\left(x^{\prime}\right)-v(x)\right|^{p} \frac{d s_{x^{\prime}}}{\left|x^{\prime}-x\right|^{n+p-2}} \\
& +\frac{1}{\varphi(z)^{2(n-1)}} \iint_{\substack{\left\{x^{\prime}, \xi^{\prime} \in \Gamma: z^{\prime} \in \delta_{1}(z), \zeta^{\prime} \in \delta_{2}(z)\right\}}}\left|v\left(x^{\prime}\right)-v\left(\xi^{\prime}\right)\right|^{p} \frac{d s_{x^{\prime}} d s_{\xi^{\prime}}}{\left|x^{\prime}-\xi^{\prime}\right|^{n+p-2}} \\
& +\frac{1}{\varphi(z)^{n-1}} \int_{\left\{\xi^{\prime} \in \Gamma: \zeta^{\prime} \in \delta_{2}(z)\right\}}\left|v\left(\xi^{\prime}\right)-v(\xi)\right|^{p} \frac{d s_{\xi^{\prime}}}{\left|\xi^{\prime}-\xi\right|^{n+p-2}} .
\end{aligned}
$$

Next we integrate the resulting inequality over $S$. Keeping (3.11)-(3.13) in mind and changing the order of integration, we arrive at (3.10). 
Remark 2. The form of the norm (3.3) shows that a linear map $T W_{p}^{1}(\Omega) \ni v \mapsto \psi v \in$ $T W_{p}^{1}(\Omega)$ is continuous if $\psi$ is Lipschitz continuous on $\partial \Omega 3$

In the sequel we need an analog of the Poincaré inequality for functions defined on surfaces. Let $\sigma$ be a measurable subset of the boundary of a domain of class $C^{0,1}$ with positive area $|\sigma|$.

Lemma 5. If $v \in L_{p}(\sigma), 1<p<\infty$, then

$$
\|v-\widetilde{v}\|_{L_{p}(\sigma)}^{p} \leq(\operatorname{diam} \sigma)^{n+p-2}|\sigma|^{-1}[v]_{p, \sigma}^{p},
$$

where $\widetilde{v}$ is the mean value of $v$ on $\sigma$ :

$$
\widetilde{v}=|\sigma|^{-1} \int_{\sigma} v(x) d s_{x}
$$

and $[\cdot]_{p, \sigma}$ is the seminorm defined in (1.1).

This assertion is proved easily with the help of Hölder's inequality.

Now we establish two lemmas concerning the space $T W_{p}^{1}(\Omega)$.

Lemma 6. If $\Omega$ is a domain with an outward peak and $v \in T W_{p}^{1}(\Omega)$, then there is a constant $c=c(p, \Omega)>0$ such that

$$
\iint_{\Gamma \times \Gamma}\left|\mu_{0}(z) v(x)-\mu_{0}(\zeta) v(\xi)\right|^{p} \chi\left(\frac{|z-\zeta|}{2 M(z, \zeta)}\right) \frac{d s_{x} d s_{\xi}}{|x-\xi|^{n+p-2}} \leq c\|v\|_{W_{p}^{1-1 / p}\left(\Gamma_{0}\right)}^{p}
$$

(we use the same notation as in (3.4)).

Proof. The left-hand side of (3.14) does not exceed the sum $c I_{1}+c I_{2}$, where

$$
\begin{aligned}
I_{1} & =\int_{\Gamma}|v(x)|^{p} d s_{x} \int_{\{\xi \in \Gamma: \zeta \in(z-2 \varphi(z), z)\}} \frac{\left|\mu_{0}(z)-\mu_{0}(\zeta)\right|^{p} d s_{\xi}}{|x-\xi|^{n+p-2}}, \\
I_{2} & =\int_{\Gamma} d s_{x} \int_{\{\xi \in \Gamma: \zeta \in(z-2 \varphi(z), z)\}} \frac{\mu_{0}(\zeta)^{p}|v(x)-v(\xi)|^{p} d s_{\xi}}{|x-\xi|^{n+p-2}} .
\end{aligned}
$$

Since $\mu_{0}(z)=\mu_{0}(\zeta)=0$ for $z, \zeta \in\left(0, z_{1}\right)$, it follows that

$$
I_{1} \leq c \int_{\left\{x \in \Gamma: z>z_{1}\right\}}|v(x)|^{p} d s_{x} \int_{\{\xi \in \Gamma: \zeta \in(z-2 \varphi(z), z)\}} \frac{d s_{\xi}}{|x-\xi|^{n-2}} .
$$

The last integral with respect to $\xi$ is not greater than $c \varphi(z)$, whence $I_{1} \leq c\|v\|_{L_{p}\left(\Gamma_{0}\right)}^{p}$. Next, since $\mu_{0}(\zeta)=0$ for $\zeta<z_{1}$, integration in $I_{2}$ is in fact only over the set $z>\zeta>z_{1}$. Therefore, $I_{2} \leq c[v]_{p, \Gamma_{0}}^{p}$, where $[\cdot]_{p, \Gamma_{0}}$ is the seminorm (1.1), and (3.14) follows.

Remark 3. In particular, for $v \in T W_{p}^{1}(\Omega)$, (3.14) implies that

$$
\left\|\mu_{0} v\right\|_{T W_{p}^{1}(\Omega)} \leq \mathrm{const} \cdot\|v\|_{W_{p}^{1-1 / p}\left(\Gamma_{0}\right)} .
$$

Lemma 7. Let $\Omega$ be the same as above, and let $v \in T W_{p}^{1}(\Omega)$ be such that $v(x)=0$ outside the set $\left\{x \in \Gamma: z \leq z_{0}\right\}$. Then $\|v\|_{T W_{p}^{1}(\Omega)} \sim|v|_{p, \Gamma}$, where $|\cdot|_{p, \Gamma}$ is the seminorm (3.4).

\footnotetext{
${ }^{3}$ To arrive at the same conclusion in the general case, it suffices to use the following fact: any Lipschitz continuous function on $\partial \Omega$ extends to a Lipschitz continuous function on $\mathbb{R}^{n}$ with the same Lipschitz constant and the same maximum of the modulus (see [9, Chapter VI, §2]). Then

$$
\begin{aligned}
\|\psi v\|_{T W_{p}^{1}(\Omega)} & =\inf \left\{\|u\|_{W_{p}^{1}(\Omega)}:\left.u\right|_{\partial \Omega}=\psi v\right\} \leq \inf \left\{\|\psi u\|_{W_{p}^{1}(\Omega)}:\left.u\right|_{\partial \Omega}=v\right\} \\
& \leq c(\psi) \inf \left\{\|u\|_{W_{p}^{1}(\Omega)}:\left.u\right|_{\partial \Omega}=v\right\}=c(\psi)\|v\|_{T W_{p}^{1}(\Omega)} .
\end{aligned}
$$
}


Proof. We use the following fact: if $w$ is a function defined on $\Gamma, w(x)=0$ for $z>z_{0}$, and $|w|_{p, \Gamma}<\infty$, then there exists a linear map $w \mapsto E w=u \in W_{p}^{1}(\Omega \cap U)$ such that $\left.u\right|_{\Gamma}=w, u(y, z)=0$ in the vicinity of $z=1$, and the estimate

$$
\|u\|_{W_{p}^{1}(\Omega \cap U)} \leq c|w|_{p, \Gamma}
$$

is valid with constant independent of $w$ (see [8, Remark 7.2/2]). Now, let $u=E v$ on $\Omega \cap U$ and $u=0$ on $\Omega \backslash U$. Then $u \in W_{p}^{1}(\Omega),\left.u\right|_{\partial \Omega}=v$, and

$$
\|u\|_{W_{p}^{1}(\Omega)} \leq c|v|_{p, \Gamma} .
$$

On the other hand, by the definition of the norm in $T W_{p}^{1}(\Omega)$, we have

$$
\|v\|_{T W_{p}^{1}(\Omega)} \leq c\|u\|_{W_{p}^{1}(\Omega)} .
$$

Hence, $\|v\|_{T W_{p}^{1}(\Omega)} \leq c|v|_{p, \Gamma}$. The reverse inequality is obvious.

\section{$\S 4$. The SPACE $T W_{p}^{1}(\Omega)^{*}$ FOR A DOMAIN WITH OUTWARD PEAK}

The theorem stated below gives a description of the space $T W_{p}^{1}(\Omega)^{*}$ dual to $T W_{p}^{1}(\Omega)$ for a domain with outward peak. Preliminarily, we introduce the space $Y_{p}(0,1)$ of functions in $L_{p, \text { loc }}(0,1)$ with finite norm

$$
\begin{aligned}
\|u\|_{Y_{p}(0,1)}= & \left(\int_{0}^{1}|u(z)|^{p} \varphi(z)^{n-1} d z\right. \\
& \left.+\iint_{\{z, \zeta \in(0,1)\}}|u(z)-u(\zeta)|^{p} \frac{M(z, \zeta)^{n-2}}{|z-\zeta|^{p}} \chi\left(\frac{|z-\zeta|}{M(z, \zeta)}\right) d z d \zeta\right)^{1 / p},
\end{aligned}
$$

where, as above, $M(z, \zeta)=\max \{\varphi(z), \varphi(\zeta)\}$, and $\chi$ is the characteristic function of the interval $(0,1)$.

Theorem 2. Let $\Omega$ be a domain with outward peak and $\left\{\mu_{k}\right\}_{k \geq 0}$ the partition of unity on $\partial \Omega \backslash\{O\}$ described in $\S 2$.

(i) Let $f \in T W_{p}^{1}(\Omega)^{*}$. Then $f$ can be represented as the sum of three terms

$$
f=\mu_{0} f+\left(1-\mu_{0}\right) \bar{f}+\left(1-\mu_{0}\right)(f-\bar{f})=f^{(1)}+f^{(2)}+f^{(3)},
$$

where each term is in $T W_{p}^{1}(\Omega)^{*}$ and the following properties are fulfilled. The support of $f^{(1)}$ lies in $\Gamma_{0}$ and $f^{(1)} \in W_{p^{\prime}}^{-1 / p^{\prime}}\left(\Gamma_{0}\right)$. The functional $f^{(2)}$, defined by

$$
T W_{p}^{1}(\Omega) \ni v \mapsto\left\langle f^{(2)}, v\right\rangle=\left\langle f,\left(1-\mu_{0}\right) \bar{v}\right\rangle,
$$

has support in the set $\left\{x \in \Gamma: z<z_{0}\right\}$, and belongs to the space $Y_{p}(0,1)^{*}$ in the sense that

$$
\left|\left\langle f^{(2)}, v\right\rangle\right| \leq \text { const } \cdot\|\bar{v}\|_{Y_{p}(0,1)} .
$$

Finally, $f^{(3)}$ has support in $\left\{x \in \Gamma: z<z_{0}\right\}$, we have

$$
\left\langle f^{(3)}, v\right\rangle=\sum_{k \geq 1}\left\langle\mu_{k}(f-\bar{f}), v\right\rangle, \quad v \in T W_{p}^{1}(\Omega),
$$

and there is a positive constant $c=c(p, \Omega)$ such that

$$
\left(\sum_{k \geq 1}\left\|\mu_{k}(f-\bar{f})\right\|_{W_{p^{\prime}}^{-1 / p^{\prime}}\left(\Gamma_{k}\right)}^{p^{\prime}}\right)^{1 / p^{\prime}} \leq c\left\|\left(1-\mu_{0}\right)(f-\bar{f})\right\|_{T W_{p}^{1}(\Omega)^{*}}
$$


(ii) Suppose that functionals $f_{k} \in W_{p^{\prime}}^{-1 / p^{\prime}}\left(\Gamma_{k}\right), k \geq 1$, are such that $\operatorname{supp} f_{k} \subset \Gamma_{k}$, $\left\langle f_{k}, v\right\rangle=0$ whenever $v \in W_{p}^{1-1 / p}\left(\Gamma_{k}\right)$ and $v(y, z)$ depends only on $z$, and moreover, we have

$$
\sum_{k \geq 1}\left\|\lambda_{k} f_{k}\right\|_{W_{p^{\prime}}^{-1 / p^{\prime}}\left(\Gamma_{k}\right)}^{p^{\prime}}<\infty
$$

Then the functional defined by

$$
T W_{p}^{1}(\Omega) \ni v \mapsto\left\langle f^{(3)}, v\right\rangle=\sum_{k \geq 1}\left\langle\lambda_{k} f_{k}, v\right\rangle
$$

is continuous, has support in $\left\{x \in \Gamma: z \leq z_{0}\right\}$, and the estimate

$$
\left\|f^{(3)}\right\|_{T W_{p}^{1}(\Omega)^{*}} \leq c\left(\sum_{k \geq 1}\left\|\lambda_{k} f_{k}\right\|_{W_{p^{\prime}}^{-1 / p^{\prime}}\left(\Gamma_{k}\right)}^{p^{\prime}}\right)^{1 / p^{\prime}}
$$

is valid with a constant depending only on $p, \Omega$. Given $h \in W_{p^{\prime}}^{-1 / p^{\prime}}\left(\Gamma_{0}\right)$ and $g \in Y_{p}(0,1)^{*}$, define $f^{(1)}=\mu_{0} h$ and

$$
\left\langle f^{(2)}, v\right\rangle=\left\langle g,\left(1-\mu_{0}\right) \bar{v}\right\rangle, \quad v \in T W_{p}^{1}(\Omega) .
$$

Then $f^{(1)}, f^{(2)} \in T W_{p}^{1}(\Omega)^{*}$, and also $f^{(1)} \in W_{p^{\prime}}^{-1 / p^{\prime}}\left(\Gamma_{0}\right)$.

Proof. (i) The fact that $f^{(1)}=\mu_{0} f \in T W_{p}^{1}(\Omega)^{*}$ follows from Remark 2. Furthermore, we have

$$
\left|\left\langle f^{(1)}, v\right\rangle\right|=\left|\left\langle f, \mu_{0} v\right\rangle\right| \leq c\|f\|_{T W_{p}^{1}(\Omega) *}\left\|\mu_{0} v\right\|_{T W_{p}^{1}(\Omega)} .
$$

Since $\left\|\mu_{0} v\right\|_{T W_{p}^{1}(\Omega)} \leq c\|v\|_{W_{p}^{1-1 / p}\left(\Gamma_{0}\right)}$ (see Remark 3 before Lemma 7$)$, we obtain $f^{(1)} \in$ $W_{p^{\prime}}^{-1 / p^{\prime}}\left(\Gamma_{0}\right)$.

Turning to $f^{(2)}$, we can write

$$
\left|\left\langle f^{(2)}, v\right\rangle\right|=\left|\left\langle f,\left(1-\mu_{0}\right) \bar{v}\right\rangle\right|,
$$

where $\bar{v}=\bar{v}(z)$ is the mean value of $\left.v\right|_{\Gamma}$, defined as in (3.1). The continuity of $f$ and Lemma 7 show that the quantity (4.7) is dominated by the expression $c(f)\left|\left(1-\mu_{0}\right) v\right|_{p, \Gamma}$ (the seminorm $|\cdot|_{p, \Gamma}$ was defined in (3.4)). Applying Lemma 3, we get a majorant for the quantity (4.7) raised to the power $p$ in the form

$$
c \iint_{S}\left|\left(1-\mu_{0}(z)\right) \bar{v}(z)-\left(1-\mu_{0}(\zeta)\right) \bar{v}(\zeta)\right|^{p} M(z, \zeta)^{n-2} \frac{d z d \zeta}{|z-\zeta|^{p}},
$$

where $S=\{(z, \zeta) \in(0,1) \times(0,1):|z-\zeta|<M(z, \zeta)\}$. Next, we dominate the expression (4.8) by the sum

$c \iint_{S} \frac{|\bar{v}(z)-\bar{v}(\zeta)|^{p}}{|z-\zeta|^{p}} M(z, \zeta)^{n-2} d z d \zeta+c \iint_{S} \frac{\left|\mu_{0}(z)-\mu_{0}(\zeta)\right|^{p}}{|z-\zeta|^{p}}|\bar{v}(z)|^{p} M(z, \zeta)^{n-2} d z d \zeta$.

The second term is not greater than

$$
c \int_{0}^{1} \bar{v}(z)^{p} \varphi(z)^{n-1} d z
$$

which does not exceed $c \int_{\Gamma}|v(x)|^{p} \varphi(z) d s_{x}$, and we arrive at inequality (4.1) with constant independent of $v$. It remains to note that the right-hand side of (4.1) is not greater than $c\|v\|_{T W_{p}^{1}(\Omega)}$ by Lemma 3 . Hence, $f^{(2)} \in T W_{p}^{1}(\Omega)^{*}$. 
Consider the term $f^{(3)}$. We observe that the relation $f^{(3)} \in T W_{p}^{1}(\Omega)^{*}$ will follow if we check that $f^{(1)}, f^{(2)} \in T W_{p}^{1}(\Omega)^{*}$. To prove $(4.2)$, for each $k=1,2, \ldots$ we select a function $v_{k} \in W_{p}^{1-1 / p}\left(\Gamma_{k}\right)$ with norm at most 1 and such that

$$
\left\|\mu_{k}(f-\bar{f})\right\|_{W_{p^{\prime}}^{-1 / p^{\prime}}\left(\Gamma_{k}\right)} \leq 2\left\langle\mu_{k}(f-\bar{f}), v_{k}\right\rangle .
$$

Let $\alpha_{k}=\left\|\mu_{k}(f-\bar{f})\right\|_{W_{p^{\prime}}^{-1 / p^{\prime}}\left(\Gamma_{k}\right)}$. For any positive integer $N$ we have

$$
\sum_{k=1}^{N} \alpha_{k}^{p^{\prime}} \leq 2 \sum_{k=1}^{N} \alpha_{k}^{p^{\prime}-1}\left\langle\mu_{k}(f-\bar{f}), v_{k}\right\rangle=2\left\langle f-\bar{f}, \sum_{k=1}^{N} \alpha_{k}^{p^{\prime}-1} \mu_{k}\left(v_{k}-\stackrel{\circ}{v}_{k}\right)\right\rangle,
$$

where $\stackrel{\circ}{v}_{k}$ is the mean value of $v_{k}$ on $\Gamma_{k}$.

In (2.12) we introduced a function $\nu_{1} \in C_{0}^{\infty}\left(z_{2}, z_{0}\right)$ such that $\mu_{1}=\left(1-\mu_{0}\right) \nu_{1}$ whereas $\mu_{k}=\left(1-\mu_{0}\right) \mu_{k}$ for $k \geq 2$. Thus, defining $\nu_{k}=\mu_{k}$ for $k=2,3, \ldots$, we rewrite (4.9) in the form

$$
\sum_{k=1}^{N} \alpha_{k}^{p^{\prime}} \leq 2\left\langle\left(1-\mu_{0}\right)(f-\bar{f}), \sum_{k=1}^{N} \alpha_{k}^{p^{\prime}-1} \nu_{k}\left(v_{k}-\stackrel{\circ}{v}_{k}\right)\right\rangle .
$$

Assuming that $\nu_{k}(z) v_{k}(x)=0$ outside $\Gamma_{k}$, we define $v(x)$ for $x \in \partial \Omega$ by

$$
v(x)=\sum_{k=1}^{N} \alpha_{k}^{p^{\prime}-1} \nu_{k}(z) w_{k}(x), \quad w_{k}(x)=v_{k}(x)-\stackrel{\circ}{v}_{k},
$$

and estimate $\|v\|_{T W_{p}^{1}(\Omega)}$. Since $\operatorname{supp} v \subset\left\{x \in \Gamma: z \leq z_{0}\right\}$, Lemma 7 shows that it suffices to bound the seminorm $|v|_{p, \Gamma}$. Let $x, \xi \in \Gamma$. We have

$$
v(x)-v(\xi)=\sum_{k=1}^{N} \alpha_{k}^{p^{\prime}-1}\left(\nu_{k}(z) w_{k}(x)-\nu_{k}(\zeta) w_{k}(\xi)\right),
$$

and moreover, for fixed $x, \xi$, the last sum contains at most four nonzero summands. Hence,

$$
|v(x)-v(\xi)|^{p} \leq c \sum_{k=1}^{N} \alpha_{k}^{\left(p^{\prime}-1\right) p}\left|\nu_{k}(z) w_{k}(x)-\nu_{k}(\zeta) w_{k}(\xi)\right|^{p}
$$

whence

$$
|v|_{p, \Gamma}^{p} \leq c \sum_{k=1}^{N} \alpha_{k}^{\left(p^{\prime}-1\right) p}\left|\nu_{k} w_{k}\right|_{p, \Gamma}^{p} .
$$

If $x \notin \Gamma_{k}$ and $\xi \notin \Gamma_{k}$, then the function

$$
\Gamma \times \Gamma \ni(x, \xi) \mapsto \nu_{k}(z) w_{k}(x)-\nu_{k}(\zeta) w_{k}(\xi)
$$

equals zero. Consequently,

$$
\begin{aligned}
\left|\nu_{k} w_{k}\right|_{p, \Gamma}^{p} \leq & c\left[\nu_{k} w_{k}\right]_{p, \Gamma_{k}}^{p} \\
& +c \int_{\Gamma_{k}}\left|\nu_{k}(z) w_{k}(x)\right|^{p} d s_{x} \int_{\left\{\xi \notin \Gamma_{k}:|z-\zeta|<M(z, \zeta)\right\}}|x-\xi|^{2-p-n} d s_{\xi},
\end{aligned}
$$

where $[\cdot]_{p, \Gamma_{k}}$ is the seminorm (1.1). Note that for $z \in \operatorname{supp} \nu_{k}$ and $\zeta \notin \Delta_{k}$ we have $|z-\zeta| \geq c \varphi\left(z_{k}\right)$ by (2.3), so that $|x-\xi| \sim \varphi\left(z_{k}\right)$. Thus, the second term on the right in (4.12) is dominated by

$$
c \varphi\left(z_{k}\right)^{1-p} \int_{\Gamma_{k}}\left|v_{k}(x)-\stackrel{\circ}{v}_{k}\right|^{p} d s_{x},
$$


with a constant independent of $k$ and $v_{k}$. Finally, by Lemma 5, the expression (4.13) does not exceed $c\left[v_{k}\right]_{p, \Gamma_{k}}^{p}$.

In order to bound the quantity $\left[\nu_{k} w_{k}\right]_{p, \Gamma_{k}}$, first we use the estimate

$$
\left[\nu_{k} w_{k}\right]_{p, \Gamma_{k}}^{p} \leq c\left[v_{k}\right]_{p, \Gamma_{k}}^{p}+c \int_{\Gamma_{k}}\left|v_{k}(x)-\stackrel{\circ}{v}_{k}\right|^{p} d s_{x} \int_{\Gamma_{k}} \frac{\left|\nu_{k}(z)-\nu_{k}(\zeta)\right|^{p}}{|x-\xi|^{n+p-2}} d s_{\xi} .
$$

By (2.3), we have

$$
\left|\nu_{k}(z)-\nu_{k}(\zeta)\right| \leq c \varphi\left(z_{k}\right)^{-1}|z-\zeta| .
$$

Therefore, the inner integral over $\Gamma_{k}$ is not greater than

$$
c \varphi\left(z_{k}\right)^{-p} \int_{\Gamma_{k}}|x-\xi|^{2-n} d s_{\xi} \leq c \varphi\left(z_{k}\right)^{1-p} .
$$

Using Lemma 5 , we dominate the second term on the right in (4.14) by $c\left[v_{k}\right]_{p, \Gamma_{k}}^{p}$.

Thus, we have established the following estimate:

$$
\left|\nu_{k} w_{k}\right|_{p, \Gamma} \leq c\left[v_{k}\right]_{p, \Gamma_{k}}
$$

with a constant $c$ independent of $k$ and $v_{k}$. Combining this estimate, (4.11), and the inequality $\left\|v_{k}\right\|_{W_{p}^{1-1 / p}\left(\Gamma_{k}\right)} \leq 1$, we see that

$$
|v|_{p, \Gamma}^{p} \leq c \sum_{k=1}^{N} \alpha_{k}^{\left(p^{\prime}-1\right) p}=c \sum_{k=1}^{N} \alpha_{k}^{p^{\prime}} .
$$

Thus the right-hand side of (4.10) does not exceed

$$
c\left\|\left(1-\mu_{0}\right)(f-\bar{f})\right\|_{T W_{p}^{1}(\Omega)^{*}}\left(\sum_{k=1}^{N} \alpha_{k}^{p^{\prime}}\right)^{1 / p} .
$$

Now, from (4.10) we deduce the estimate

$$
\sum_{k=1}^{N} \alpha_{k}^{p^{\prime}} \leq c\left\|\left(1-\mu_{0}\right)(f-\bar{f})\right\|_{T W_{p}^{1}(\Omega)^{*}}\left(\sum_{k=1}^{N} \alpha_{k}^{p^{\prime}}\right)^{1 / p}
$$

with constant independent of $\alpha_{k}=\left\|\mu_{k}(f-\bar{f})\right\|_{W_{p^{\prime}}^{-1 / p^{\prime}}\left(\Gamma_{k}\right)}$ and $N$. Hence, (4.2) follows.

(ii) Let $v \in T W_{p}^{1}(\Omega)$. Then

$$
\begin{aligned}
\sum_{k \geq 1}\left|\left\langle\lambda_{k} f_{k}, v\right\rangle\right| & \leq \sum_{k \geq 1}\left|\left\langle\lambda_{k} f_{k}, \sum_{|k-i| \leq 1} \mu_{i}\left(v-\stackrel{\circ}{v}_{k}\right)\right\rangle\right| \\
& \leq \sum_{k \geq 1} \sum_{|k-i| \leq 1}\left\|\lambda_{k} f_{k}\right\|_{W_{p^{\prime}}^{-1 / p^{\prime}}\left(\Gamma_{k}\right)}\left\|\mu_{i}\left(v-\stackrel{\circ}{v}_{k}\right)\right\|_{W_{p}^{1-1 / p}\left(\Gamma_{k}\right)},
\end{aligned}
$$

where $\stackrel{\circ}{v}_{k}$ is the mean value of $v$ on $\Gamma_{k}$. Clearly,

$$
\left\|\mu_{i}\left(v-\stackrel{\circ}{v}_{k}\right)\right\|_{W_{p}^{1-1 / p}\left(\Gamma_{k}\right)} \leq\left\|v-\stackrel{\circ}{v}_{k}\right\|_{L_{p}\left(\Gamma_{k}\right)}+\left[\mu_{i}\left(v-\stackrel{\circ}{v}_{k}\right)\right]_{p, \Gamma_{k}} .
$$

We use Lemma 5 to bound the first term on the right:

$$
\left\|v-\stackrel{\circ}{v}_{k}\right\|_{L_{p}\left(\Gamma_{k}\right)} \leq c \varphi\left(z_{k}\right)^{1-1 / p}[v]_{p, \Gamma_{k}} .
$$

Furthermore,

$$
\left[\mu_{i}\left(v-\stackrel{\circ}{v}_{k}\right)\right]_{p, \Gamma_{k}}^{p} \leq c[v]_{p, \Gamma_{k}}^{p}+c \int_{\Gamma_{k}}\left|v(x)-\stackrel{\circ}{v}_{k}\right|^{p} d s_{x} \int_{\Gamma_{k}} \frac{\left|\mu_{i}(z)-\mu_{i}(\zeta)\right|^{p}}{|x-\xi|^{n+p-2}} d s_{\xi} .
$$

Since $|k-i| \leq 1$, we have $\left|\mu_{i}(z)-\mu_{i}(\zeta)\right| \leq c \varphi\left(z_{k}\right)^{-1}|z-\zeta|$, and the second term on the right in $(4.16)$ is dominated by the first summand exactly in the same way as the 
second term on the right in (4.14) is dominated by the first. So, we have established the estimate

$$
\left\|\mu_{i}\left(v-\stackrel{\circ}{v}_{k}\right)\right\|_{W_{p}^{1-1 / p}\left(\Gamma_{k}\right)} \leq c[v]_{p, \Gamma_{k}} .
$$

Now, from (4.15) it follows that

$$
\begin{aligned}
\sum_{k \geq 1}\left|\left\langle\lambda_{k} f_{k}, v\right\rangle\right| & \leq c \sum_{k \geq 1}\left\|\lambda_{k} f_{k}\right\|_{W_{p^{\prime}}^{-1 / p^{\prime}}\left(\Gamma_{k}\right)}[v]_{p, \Gamma_{k}} \\
& \leq c\left(\sum_{k \geq 1}\left\|\lambda_{k} f_{k}\right\|_{W_{p^{\prime}}^{p^{\prime}}}^{p^{\prime} / p^{\prime}\left(\Gamma_{k}\right)}\right)^{1 / p^{\prime}}\left(\sum_{k \geq 1}[v]_{p, \Gamma_{k}}^{p}\right)^{1 / p} .
\end{aligned}
$$

Note that for $x, \xi \in \Gamma_{k}$ we have $|z-\zeta|<2 M(z, \zeta)$. Therefore,

$$
\sum_{k \geq 1}[v]_{p, \Gamma_{k}}^{p} \leq \iint_{\{x, \xi \in \Gamma:|\zeta-z|<2 M(z, \zeta)\}}|v(x)-v(\xi)|^{p} \frac{d s_{x} d s_{\xi}}{|x-\xi|^{n+p-2}} .
$$

By Lemmas 4 and 6, the right-hand side of the last estimate is not greater than

$$
c\left|\left(1-\mu_{0}\right) v\right|_{p, \Gamma}^{p}+c\left\|\mu_{0} v\right\|_{W_{p}^{1-1 / p}\left(\Gamma_{0}\right)}^{p},
$$

which does not exceed $c\|v\|_{T W_{p}^{1}(\Omega)}^{p}$ by Remark 2. Now, (4.17) implies that the functional (4.4) is well defined and continuous, and its norm is subject to (4.5).

The fact that $f^{(1)} \in W_{p^{\prime}}^{-1 / p^{\prime}}\left(\Gamma_{0}\right)$ follows from Gagliardo's theorem and Remark 2, and since $T W_{p}^{1}(\Omega) \subset W_{p}^{1-1 / p}\left(\Gamma_{0}\right)$, we also have $f^{(1)} \in T W_{p}^{1}(\Omega)^{*}$.

The proof of the continuity on $T W_{p}^{1}(\Omega)$ of the functional (4.6) under the condition $g \in Y_{p}(0,1)^{*}$ is carried out in the same way as in statement (i). This completes the proof of Theorem 2 .

Now we state some corollaries to the above theorem. The first follows from the definition of the solution of the Neumann problem considered in $\S 1$.

Corollary 4. Let $\Omega$ be a domain with outward peak, and let $f: W_{p}^{1}(\Omega) \rightarrow \mathbb{R}^{1}$ be the sum of three functionals $f^{(1)}, f^{(2)}, f^{(3)}$ described in statement (ii) of Theorem 2 . Then the Neumann problem (1.2), (1.3) is uniquely solvable.

Corollary 5. Let $f$ be a linear functional defined on $T W_{p}^{1}(\Omega)$, and let $\bar{f}$ be defined on the set of functions with support in $\Gamma$ by

$$
\langle\bar{f}, v\rangle=\langle f, \bar{v}\rangle .
$$

Then the functional $\left(1-\mu_{0}\right)(f-\bar{f})$ is in $T W_{p}^{1}(\Omega)^{*}$ if and only if

$$
\sum_{k \geq 1}\left\|\mu_{k}(f-\bar{f})\right\|_{W_{p^{\prime}}^{-1 / p^{\prime}}\left(\Gamma_{k}\right)}^{p^{\prime}}<\infty .
$$

Moreover, the following equivalence relation holds true:

$$
\left(\sum_{k \geq 1}\left\|\mu_{k}(f-\bar{f})\right\|_{W_{p^{\prime}}^{-1 / p^{\prime}}\left(\Gamma_{k}\right)}^{p^{\prime}}\right)^{1 / p^{\prime}} \sim\left\|\left(1-\mu_{0}\right)(f-\bar{f})\right\|_{T W_{p}^{1}(\Omega)^{*}} .
$$

The constants in this relation depend only on $p$ and $\Omega$.

The proof is similar to that of Corollary 2. It suffices to replace the references to Theorem 1 by those to Theorem 2 .

Corollary 5 makes it possible to establish the continuity of the linear map

$$
T W_{p}^{1}(\Omega) \ni v \mapsto v-\bar{v} \in L_{q}(\Gamma)
$$


with the maximal Sobolev exponent $q$, and also to prove that $f-\bar{f} \in T W_{p}^{1}(\Omega)^{*}$ for $f \in L_{q^{\prime}}(\partial \Omega)$ with $q^{\prime}=q /(q-1)$.

Corollary 6. Let $q=(n-1) p /(n-p)$ for $p<n, q \in[p, \infty)$ for $p=n$, and $q=\infty$ for $p>n$. Also, let $q^{\prime-1}+q^{-1}=1$. If $f \in L_{q^{\prime}}(\partial \Omega)$, then the functional

$$
T W_{p}^{1}(\Omega) \ni v \mapsto\left\langle\left(1-\mu_{0}\right)(f-\bar{f}), v\right\rangle=\int_{\Gamma} f(x)(v(x)-\bar{v}(z))\left(1-\mu_{0}(z)\right) d s_{x}
$$

belongs to $T W_{p}^{1}(\Omega)^{*}$, and the estimate

$$
\left\|\left(1-\mu_{0}\right)(f-\bar{f})\right\|_{T W_{p}^{1}(\Omega)^{*}} \leq c\|f-\bar{f}\|_{L_{q^{\prime}}(\Gamma)}
$$

is valid with constant independent of $f$. Furthermore, for all $v \in T W_{p}^{1}(\Omega)$ we have

$$
\|v-\bar{v}\|_{L_{q}(\Gamma)} \leq c\|v\|_{T W_{p}^{1}(\Omega)}
$$

with constant independent of $v$.

Proof. First, we bound the sum on the left in (4.18). If $v \in W_{p}^{1-1 / p}\left(\Gamma_{k}\right)$, then, by Hölder's inequality,

$$
\begin{aligned}
\left|\left\langle\mu_{k}(f-\bar{f}), v\right\rangle\right| & =\left|\int_{\Gamma_{k}} \mu_{k}(f-\bar{f})\left(v-\stackrel{\circ}{v}_{k}\right) d s_{x}\right| \\
& \leq\|f-\bar{f}\|_{L_{q^{\prime}}\left(\Gamma_{k}\right)}\left\|\mu_{k}\left(v-\stackrel{\circ}{v}_{k}\right)\right\|_{L_{q}\left(\Gamma_{k}\right)},
\end{aligned}
$$

where, as before, $\stackrel{\circ}{v}_{k}$ is the mean value of $v$ on $\Gamma_{k}$. Clearly,

$$
\Omega_{k}=\left\{(y, z): z \in\left(z_{k+1}, z_{k-1}\right), y / \varphi(z) \in \omega\right\}
$$

is a domain of class $C^{0,1}$. Hence, by the Sobolev theorem, for any $u \in W_{p}^{1-1 / p}\left(\partial \Omega_{k}\right)$ we have

$$
\|u\|_{L_{q}\left(\partial \Omega_{k}\right)} \leq c \varphi\left(z_{k}\right)^{\frac{n-1}{q}-\frac{n-1}{p}}\|u\|_{L_{p}\left(\partial \Omega_{k}\right)}+c \varphi\left(z_{k}\right)^{1+\frac{n-1}{q}-\frac{n}{p}}[u]_{p, \partial \Omega_{k}} .
$$

First, we consider the case where $p<n$. Then

$$
\|u\|_{L_{q}\left(\partial \Omega_{k}\right)} \leq c \varphi\left(z_{k}\right)^{-1 / p^{\prime}}\|u\|_{L_{p}\left(\partial \Omega_{k}\right)}+c[u]_{p, \partial \Omega_{k}} .
$$

Here $[\cdot]_{p, \partial \Omega_{k}}$ is the seminorm (1.1). Let $u=\mu_{k}\left(v-\stackrel{\circ}{v}_{k}\right)$. We insert $u$ into the above inequality, assuming that $u=0$ on $\partial \Omega_{k} \backslash \Gamma_{k}$, and use Lemma 5 to bound the first term on the right. This results in

$$
\left\|\mu_{k}\left(v-\stackrel{\circ}{v}_{k}\right)\right\|_{L_{q}\left(\Gamma_{k}\right)} \leq c[v]_{p, \Gamma_{k}}+c\left[\mu_{k}\left(v-\stackrel{\circ}{v}_{k}\right)\right]_{p, \partial \Omega_{k}} .
$$

To bound the second term, we write

$$
\begin{aligned}
{\left[\mu_{k}\left(v-\stackrel{\circ}{v}_{k}\right)\right]_{p, \partial \Omega_{k}}^{p} } & \leq c\left[\mu_{k}\left(v-\stackrel{\circ}{v}_{k}\right)\right]_{p, \Gamma_{k}}^{p} \\
& +c \int_{\Gamma_{k}}\left|\mu_{k}(z)\left(v(x)-\stackrel{\circ}{v}_{k}\right)\right|^{p} d s_{x} \int_{\partial \Omega_{k} \backslash \Gamma_{k}}|x-\xi|^{2-n-p} d s_{\xi} .
\end{aligned}
$$

If $x=(y, z) \in \Gamma_{k}, z \in \operatorname{supp} \mu_{k}$ and $\xi \notin \Gamma_{k}$, then $|x-\xi| \geq c \varphi\left(z_{k}\right)$ by (2.3). Therefore, the last term in (4.23) is not greater than

$$
c \varphi\left(z_{k}\right)^{1-p} \int_{\Gamma_{k}}\left|v(x)-\stackrel{\circ}{v}_{k}\right|^{p} d s_{x}
$$

which does not exceed $c[v]_{p, \Gamma_{k}}^{p}$ in view of Lemma 5. Next, we dominate the expression $\left[\mu_{k}\left(v-\stackrel{\circ}{v}_{k}\right]_{p, \Gamma_{k}}^{p}\right.$ by the right-hand side of (4.14), where $v_{k}(x)$ should be replaced with $v(x)$ and $\nu_{k}$ with $\mu_{k}$. Now the same argument as in Theorem 2 leads to the estimate

$$
\left[\mu_{k}\left(v-\stackrel{\circ}{v}_{k}\right)\right]_{p, \Gamma_{k}} \leq c[v]_{p, \Gamma_{k}} .
$$


Combining this with inequalities (4.22) and (4.23), we get

$$
\left\|\mu_{k}\left(v-\stackrel{\circ}{v}_{k}\right)\right\|_{L_{q}\left(\Gamma_{k}\right)} \leq c[v]_{p, \Gamma_{k}} .
$$

A similar argument for $p \geq n$ shows that (4.24) is also true.

Inequalities (4.21) and (4.24) imply that

$$
\left\|\mu_{k}(f-\bar{f})\right\|_{W_{p^{\prime}}^{-1 / p^{\prime}}\left(\Gamma_{k}\right)} \leq c\|f-\bar{f}\|_{L_{q^{\prime}}\left(\Gamma_{k}\right)},
$$

whence

$$
\left(\sum_{k \geq 1}\left\|\mu_{k}(f-\bar{f})\right\|_{W_{p^{\prime}}^{-1 / p^{\prime}}\left(\Gamma_{k}\right)}^{p^{\prime}}\right)^{1 / p^{\prime}} \leq c\left(\sum_{k \geq 1}\|f-\bar{f}\|_{L_{q^{\prime}}\left(\Gamma_{k}\right)}^{p^{\prime}}\right)^{1 / p^{\prime}} .
$$

Applying the algebraic inequality (2.22) in the same way as in Corollary 3, we dominate the left-hand side of (4.25) by $c\|f-\bar{f}\|_{L_{q^{\prime}}(\Gamma)}$. Now, (4.19) follows from Corollary 5 .

Turning to the proof of (4.20), we note that

$$
\left\langle\left(1-\mu_{0}\right) f, v-\bar{v}\right\rangle=\left\langle\left(1-\mu_{0}\right) f-\bar{f}, v\right\rangle
$$

and $\|\bar{f}\|_{L_{q^{\prime}}(\Gamma)} \leq c\|f\|_{L_{q^{\prime}}(\Gamma)}$. Hence, (4.19) implies the estimate

$$
\left|\left\langle f,\left(1-\mu_{0}\right)(v-\bar{v})\right\rangle\right| \leq c\|f\|_{L_{q^{\prime}}(\Gamma)}\|v\|_{T W_{p}^{1}(\Omega)}
$$

for all $f \in L_{q^{\prime}}(\Gamma)$ and $v \in T W_{p}^{1}(\Omega)$. Let $V$ denote the unit ball in $T W_{p}^{1}(\Omega)$. For $v \in V$, we define

$$
L_{q^{\prime}}(\Gamma) \ni f \mapsto F_{v}(f)=\left\langle f,\left(1-\mu_{0}\right)(v-\bar{v})\right\rangle=\int_{\Gamma} f(x)\left(1-\mu_{0}(z)\right)(v(x)-\bar{v}(z)) d s_{x} .
$$

By (4.26), the functionals $F_{v}(\cdot)$ are continuous and pointwise bounded; hence, their norms are bounded uniformly in $v \in V$. This means that

$$
\left\|\left(1-\mu_{0}\right)(v-\bar{v})\right\|_{L_{q}(\Gamma)} \leq \text { const }\|v\|_{T W_{p}^{1}(\Omega)}
$$

for all $v \in T W_{p}^{1}(\Omega)$.

To complete the proof of (4.20), we check the inequality

$$
\left\|\mu_{0}(v-\bar{v})\right\|_{L_{q}(\Gamma)} \leq \mathrm{const} \cdot\|v\|_{T W_{p}^{1}(\Omega)} .
$$

Indeed, (3.1) and Hölder's inequality show that

$$
|\bar{v}(z)|^{q} \varphi(z)^{n-2}|\gamma| \leq \int_{y \in \varphi(z) \gamma}|v(y, z)|^{q} d \gamma(y) .
$$

Integration over $z \in\left(z_{1}, 1\right)$ yields

$$
\left\|\mu_{0} \bar{v}\right\|_{L_{q}\left(\Gamma \cap \Gamma_{0}\right)}^{q} \leq c\left\|\mu_{0} v\right\|_{L_{q}\left(\Gamma \cap \Gamma_{0}\right)}^{q} .
$$

It remains to observe that, by the Sobolev theorem, the norm on the right does not exceed $c\left\|\mu_{0} v\right\|_{W_{p}^{1-1 / p}\left(\Gamma_{0}\right)}$, which is not greater than $\|v\|_{T W_{p}^{1}(\Omega)}$ by Remark 2. The proof of Corollary 6 is complete.

We conclude this section with an assertion similar to Proposition 1.

Proposition 2. Let $1 \leq q \leq p(n-1) /(n-p)$ for $p<n, q \in[1, \infty)$ for $p=n$, and $1 \leq q \leq \infty$ for $p>n$. Also, let $q^{-1}+q^{\prime-1}=1$. If $\Omega \subset \mathbb{R}^{n}$ is a domain with outward peak, then the following statements are equivalent.

(A) The Neumann problem (1.2), (1.3) is solvable for all $f \in L_{q^{\prime}}(\partial \Omega)$.

(B) The space $T W_{p}^{1}(\Omega)$ is embedded in $L_{q}(\partial \Omega)$ continuously.

(C) The functional $T W_{p}^{1}(\Omega) \ni v \mapsto \int_{\partial \Omega} f v d s_{x}$ is continuous for all $f \in L_{q^{\prime}}(\partial \Omega)$.

(D) The map $T W_{p}^{1}(\Omega) \ni v \mapsto \bar{v} \in L_{q}(\Gamma)$ is continuous. 
(E) The space $Y_{p}(0,1)$ is embedded continuously in a weighted $L_{q}$ with the norm

$$
u \mapsto\left(\int_{0}^{1}|u(z)|^{q} \varphi(z)^{n-2} d z\right)^{1 / q} .
$$

Proof. (A) $\Rightarrow(\mathrm{B})$. Repeating the argument of the proof of $(\mathrm{A}) \Rightarrow(\mathrm{B})$ in Proposition 1, we obtain

$$
\|v\|_{L_{q}(\partial \Omega)} \leq \mathrm{const}\|v\|_{W_{p}^{1}(\Omega)}
$$

for all $v$ in the set $W_{p}^{1}(\Omega) \cap L_{\infty}(\Omega) \cap C^{\infty}(\Omega)$. Since this set is dense in $W_{p}^{1}(\Omega)$, the above estimate is valid for all $v \in W_{p}^{1}(\Omega)$. Thus, the restriction operator $\left.W_{p}^{1}(\Omega) \ni v \mapsto v\right|_{\partial \Omega} \in$ $L_{q}(\partial \Omega)$ is continuous, whence $T W_{p}^{1}(\Omega)$ is embedded in $L_{q}(\partial \Omega)$ continuously.

$(\mathrm{B}) \Rightarrow(\mathrm{C})$. Suppose $f \in L_{q^{\prime}}(\partial \Omega)$ and $v \in T W_{p}^{1}(\Omega)$. Since $T W_{p}^{1}(\Omega)$ is embedded in $L_{q}(\partial \Omega)$ continuously, we have

$$
\left|\int_{\partial \Omega} f v d s_{x}\right| \leq\|f\|_{L_{q^{\prime}}(\partial \Omega)}\|v\|_{L_{q}(\partial \Omega)} \leq c\|f\|_{L_{q^{\prime}}(\partial \Omega)}\|v\|_{T W_{p}^{1}(\Omega)},
$$

and the result follows.

$(\mathrm{C}) \Rightarrow(\mathrm{A})$. As was shown in $\S 1$, the continuity of the functional

$$
T W_{p}^{1}(\Omega) \ni v \mapsto \int_{\partial \Omega} f v d s_{x}
$$

implies the solvability of the Neumann problem.

The equivalence of (B) and (D) follows from Corollary 6 .

(D) $\Rightarrow(\mathrm{E})$. Let $u \in Y_{p}(0,1)$. Also, let $\lambda \in C^{\infty}(0,1)$,

$$
0 \leq \lambda \leq 1,\left.\quad \lambda\right|_{\left(0, z_{2}\right)}=1,\left.\quad \lambda\right|_{\left(z_{1}, 1\right)}=0 .
$$

Let $v \in T W_{p}^{1}(\Omega)$ be defined by $v(x)=\lambda(z) u(z)$ for $x \in \Gamma, v=0$ on $\partial \Omega \backslash \Gamma$. By Lemma 3 , the inequality $\|\bar{v}\|_{L_{q}(\Gamma)} \leq c\|v\|_{T W_{p}^{1}(\Omega)}$ is equivalent to

$$
\left(\int_{0}^{1}|\lambda(z) u(z)|^{q} \varphi(z)^{n-2} d z\right)^{1 / q} \leq c\|\lambda u\|_{Y_{p}(0,1)}
$$

The argument used in Theorem 2 to prove (4.1) applies again, showing that the righthand side of the last inequality does not exceed $c(\lambda)\|u\|_{Y_{p}(0,1)}$. Thus,

$$
\|\lambda u\|_{L_{q}(\Gamma)} \leq c\|u\|_{Y_{p}(0,1)} .
$$

Next, it is obvious that

$$
\|(1-\lambda) u\|_{L_{q}(\Gamma)} \leq c\left(\int_{z_{2}}^{1}|u(z)|^{q} d z\right)^{1 / q} .
$$

We have $1-n / p+(n-1) / q \geq 0$ by assumption, whence $1-2 / p+1 / q>0$ for $q \geq p$, so that the space $W_{p}^{1-1 / p}\left(z_{2}, 1\right)$ is embedded in $L_{q}\left(z_{2}, 1\right)$ continuously (see, e.g., [11, $\left.\S 16\right]$ ). For $q<p$, the continuity of the same embedding follows from Hölder's inequality. Hence,

$$
\left(\int_{0}^{1}|(1-\lambda(z)) u(z)|^{q} \varphi(z)^{n-2} d z\right)^{1 / q} \leq c\|u\|_{W_{p}^{1-1 / p}\left(z_{2}, 1\right)} \leq c\|u\|_{Y_{p}(0,1)} .
$$

Combining this with (4.27), we arrive at (E).

$(\mathrm{E}) \Rightarrow(\mathrm{D})$. Let $v \in T W_{p}^{1}(\Omega)$. Lemma 3 says that $\|\bar{v}\|_{Y_{p}(0,1)} \leq c\|v\|_{T W_{p}^{1}(\Omega)}$. Therefore,

$$
\left(\int_{0}^{1}|\bar{v}(z)|^{q} \varphi(z)^{n-2} d z\right)^{1 / q} \leq c\|v\|_{T W_{p}^{1}(\Omega)}
$$


which means that the map $T W_{p}^{1}(\Omega) \ni v \mapsto \bar{v} \in L_{q}(\Gamma)$ is continuous. The proof of Proposition 2 is complete.

Remark 4. By using the results of [10, the last assertion can be supplemented in the following way. It turns out that every statement $(\mathrm{A})-(\mathrm{E})$ above is equivalent to the inequality

$$
\int_{0}^{1}\left[\int_{0}^{z} \varphi(t)^{n-2} d t\left(\int_{z}^{1} \frac{d t}{\varphi(t)^{\frac{n-1}{p-1}}}\right)^{q-1}\right]^{\frac{p}{p-q}} \frac{d z}{\varphi(z)^{\frac{n-1}{p-1}}}<\infty
$$

for $q<p$, and to the inequality

$$
\sup _{r \in(0,1)}\left(\int_{0}^{r} \varphi(z)^{n-2} d z\right)^{1 / q}\left(\int_{r}^{1} \varphi(z)^{\frac{1-n}{p-1}} d z\right)^{(p-1) / p}<\infty
$$

for $q \geq p$.

Example. Consider a power cusp

$$
\Omega=\left\{(y, z) \in \mathbb{R}^{n}: z \in(0,1),|y|<c z^{\lambda}\right\}, \quad \lambda>1 .
$$

The Neumann problem (1.2), (1.3) is solvable for this domain for all $f \in L_{q^{\prime}}(\partial \Omega)$ in the following cases:

1) $p \geq 1+\lambda(n-1)$ and $q \in[1, p)$;

2) $p<1+\lambda(n-1)$ and $1 \leq q<p \min \{1,(1+\lambda(n-2)) /(1+\lambda(n-1)-p)\}$;

3) $p>1+\lambda(n-1)$ and $p \leq q \leq \infty$;

4) $p=1+\lambda(n-1)$ and $p \leq q<\infty$;

5) $1<p<1+\lambda(n-1)$ and $p \leq q \leq(\lambda(n-2)+1) p /(1+\lambda(n-1)-p)$.

\section{§5. A DOMAIN WITH INWARD PEAK}

In this section we describe the spaces dual to $W_{p}^{1}$ and $T W_{p}^{1}$ for a domain with inward peak. Let $\Omega \subset \mathbb{R}^{n}$ be a domain with outward peak for $n>2$, as before. Then its complement $G=\mathbb{R}^{n} \backslash \bar{\Omega}$ is a multidimensional domain with inner peak. By the Jones theorem [12, there is a continuous linear extension operator $E: W_{p}^{1}(G) \rightarrow W_{p}^{1}\left(\mathbb{R}^{n}\right)$. Consequently, the space $W_{p}^{1}(G)^{*}$ is a subspace of $W_{p}^{1}\left(\mathbb{R}^{n}\right)^{*}$, and the linear map

$$
W_{p}^{1}\left(\mathbb{R}^{n}\right)^{*} \ni \mathcal{F} \mapsto F \in W_{p}^{1}(G)^{*}
$$

given by $\langle F, v\rangle=\langle\mathcal{F}, E v\rangle$ is a continuous projection, so that a description of the space $W_{p}^{1}(G)^{*}$ is reduced to that of the standard class $W_{p}^{1}\left(\mathbb{R}^{n}\right)^{*}$.

We turn to a description of the space $T W_{p}^{1}(G)^{*}$. In accordance with [7] (see also [8. Chapter 7]), the space $T W_{p}^{1}(G)$ is characterized in different ways for $p \in(1, n-1)$, $p=n-1$, and $p>n-1$. Namely, for $1<p<n-1$, the norm in $T W_{p}^{1}(G)$ is equivalent to the norm

$$
\left(\|v\|_{W_{p}^{1-1 / p}\left(\Gamma_{0}\right)}^{p}+\int_{\Gamma}|v(x)|^{p} \varphi(z)^{1-p} d s_{x}+|v|_{p, \Gamma}^{p}\right)^{1 / p}
$$

where $|\cdot|_{p, \Gamma}$ is the seminorm defined in (3.4). For $p=n-1>1$ we have

$$
\|v\|_{T W_{p}^{1}(G)} \sim\left\{\|v\|_{W_{p}^{1-1 / p}\left(\Gamma_{0}\right)}^{p}+|v|_{p, \Gamma}^{p}+\int_{\Gamma} \frac{|v(x)|^{p} d s_{x}}{(\varphi(z) \log (z / \varphi(z)))^{p-1}}+\|v\|_{p, \Gamma}^{p}\right\}^{1 / p},
$$

where

$$
\|v\|_{p, \Gamma}=\left\{\iint_{S}|v(x)-v(\xi)|^{p} \frac{M(z, \zeta)^{2-2 p} d s_{x} d s_{\xi}}{|x-\xi|(1+\log (|x-\xi| / M(z, \zeta)))^{p}}\right\}^{1 / p} .
$$


Here integration is taken over the set

$$
S=\{x, \xi \in \Gamma:|z-\zeta|>M(z, \zeta), 1 / 2<z / \zeta<2\}
$$

we have used the same notation as in (3.4), and for $p=n-1$ we require additionally that $\left.\varphi^{\prime}(z)=O(\varphi(z) / z)\right)$ as $z \rightarrow 0$, which implies, in particular, that $\varphi(z) \sim \varphi(\zeta)$ if $2^{-1} \leq z \zeta^{-1} \leq 2$.

Finally, for $p>n-1$ the norm in $T W_{p}^{1}(G)$ is equivalent to

$$
\left\{\|v\|_{W_{p}^{1-1 / p}\left(\Gamma_{0}\right)}^{p}+|v|_{p, \Gamma}^{p}+\int_{\Gamma}|v(x)|^{p} \varphi(z)^{2-n} d s_{x}+(v)_{p, \Gamma}^{p}\right\}^{1 / p},
$$

where

$$
(v)_{p, \Gamma}=\left\{\iint_{\Gamma \times \Gamma}|v(x)-v(\xi)|^{p} \frac{(\varphi(z) \varphi(\zeta))^{2-n}}{|x-\xi|^{p+2-n}} \chi\left(\frac{M(z, \zeta)}{|z-\zeta|}\right) d s_{x} d s_{\xi}\right\}^{1 / p} .
$$

Each of the norms mentioned above induces a norm in $Y_{p}(0,1)$, namely, the restriction of the corresponding norm in $T W_{p}^{1}(G)$ to the subspace of functions supported in $\Gamma$ and depending only on the variable $z$. Now we write out these norms. If $p \in(1, n-1)$, then

$$
\begin{aligned}
\|u\|_{Y_{p}(0,1)}= & \left(\int_{0}^{1}|u(z)|^{p} \frac{d z}{\varphi(z)^{p+1-n}}\right. \\
& \left.+\iint_{\{z, \zeta \in(0,1)\}}|u(z)-u(\zeta)|^{p} \frac{M(z, \zeta)^{n-2}}{|z-\zeta|^{p}} \chi\left(\frac{|z-\zeta|}{M(z, \zeta)}\right) d z d \zeta\right)^{\frac{1}{p}}
\end{aligned}
$$

For $p=n-1$ we may set

$$
\|u\|_{Y_{p}(0,1)}=\left(\int_{0}^{1} \frac{|u(z)|^{p} d z}{(\log (z / \varphi(z)))^{p-1}}+\int_{0}^{1} \int_{0}^{1} \frac{|u(z)-u(\zeta)|^{p}}{|z-\zeta|} \sigma(z, \zeta) d z d \zeta\right)^{\frac{1}{p}}
$$

where

$$
\sigma(z, \zeta)=\chi_{(1 / 2,2)}(z / \zeta) Q\left(|z-\zeta|(M(z, \zeta))^{-1}\right)
$$

$\chi_{(1 / 2,2)}$ is the characteristic function of the interval $(1 / 2,2)$, and

$$
Q(t)= \begin{cases}t^{1-p} & \text { if } t \in(0,1) \\ (\log (e t))^{-p} & \text { if } t>1\end{cases}
$$

In case $p>n-1$, one may write

$$
\|u\|_{Y_{p}(0,1)}=\left(\int_{0}^{1}|u(z)|^{p} d z+\int_{0}^{1} \int_{0}^{1} \frac{|u(z)-u(\zeta)|^{p}}{|z-\zeta|^{p+2-n}} \max \left\{\frac{M(z, \zeta)^{n-2}}{|z-\zeta|^{n-2}}, 1\right\} d z d \zeta\right)^{\frac{1}{p}} .
$$

The theorem stated below gives a description of the space $T W_{p}^{1}(G)^{*}$ for a domain with inward peak. The constants appearing in the theorem depend only on $p$ and the domain.

Theorem 3. Let $\Omega$ be a domain with outward peak, let $G=\mathbb{R}^{n} \backslash \bar{\Omega}$, and let $\left\{\mu_{k}\right\}_{k \geq 0}$ be the partition of unity occurring in Theorem 2.

(i) Any functional $f$ belonging to $T W_{p}^{1}(G)^{*}$ can be represented as the sum

$$
f=\mu_{0} f+\left(1-\mu_{0}\right) \bar{f}+\left(1-\mu_{0}\right)(f-\bar{f}),
$$

where each summand on the right belongs to the same space. Furthermore, the first summand is in $W_{p^{\prime}}^{-1 / p^{\prime}}\left(\Gamma_{0}\right)$ and has support in $\Gamma_{0}$. The second summand is supported in $\left\{x \in \Gamma: z \leq z_{0}\right\}$ and belongs to $Y_{p}(0,1)^{*}$ in the sense that

$$
\left|\left\langle f^{(2)}, v\right\rangle\right| \leq \mathrm{const} \cdot\|\bar{v}\|_{Y_{p}(0,1)} .
$$


The third summand can be represented in the form

$$
\left(1-\mu_{0}\right)(f-\bar{f})=\sum_{k \geq 1} \mu_{k}(f-\bar{f})
$$

and we have

$$
\left(\sum_{k \geq 1}\left\|\mu_{k}(f-\bar{f})\right\|_{W_{p^{\prime}}^{-1 / p^{\prime}}\left(\Gamma_{k}\right)}^{p^{\prime}}\right)^{1 / p^{\prime}} \leq c\left\|\left(1-\mu_{0}\right)(f-\bar{f})\right\|_{T W_{p}^{1}(G)^{*}} .
$$

(ii) Suppose that $f_{k} \in W_{p^{\prime}}^{-1 / p^{\prime}}\left(\Gamma_{k}\right), k \geq 1$, satisfy the hypotheses of statement (ii) of Theorem 2. Let $g \in Y_{p}(0,1)^{*}$ and $h \in W_{p^{\prime}}^{-1 / p^{\prime}}\left(\Gamma_{0}\right)$. Define $f^{(1)}=\mu_{0} h$,

$$
\begin{aligned}
T W_{p}^{1}(G) \ni v & \mapsto\left\langle f^{(2)}, v\right\rangle=\left\langle g,\left(1-\mu_{0}\right) \bar{v}\right\rangle, \\
\left.T W_{p}^{1}(G)\right) \ni v & \mapsto\left\langle f^{(3)}, v\right\rangle=\sum_{k \geq 1}\left\langle\lambda_{k} f_{k}, v\right\rangle .
\end{aligned}
$$

Then $f^{(j)}$ is continuous on $T W_{p}^{1}(G), j=1,2,3$. Furthermore, $f^{(1)} \in W_{p^{\prime}}^{-1 / p^{\prime}}\left(\Gamma_{0}\right)$, and we have

$$
\left\|f^{(3)}\right\|_{T W_{p}^{1}(G)^{*}} \leq c\left(\sum_{k \geq 1}\left\|\lambda_{k} f_{k}\right\|_{W_{p^{\prime}}^{-1 / p^{\prime}}\left(\Gamma_{k}\right)}^{p^{\prime}}\right)^{1 / p^{\prime}} .
$$

Before proceeding to the proof of the theorem, we separately establish some estimates for norms (and seminorms) involved in $\|\cdot\|_{T W_{p}^{1}(G)}$.

Lemma 8. If $v \in T W_{p}^{1}(G)$, then

$$
\left\|\mu_{0} v\right\|_{T W_{p}^{1}(G)} \leq \text { const } \cdot\|v\|_{W_{p}^{1-1 / p}\left(\Gamma_{0}\right)} .
$$

Proof. We need to verify the estimates

$$
\begin{aligned}
\left|\mu_{0} v\right|_{p, \Gamma}^{p} \leq c\|v\|_{W_{p}^{1-1 / p}\left(\Gamma_{0}\right)}^{p}, & p \in(1, \infty), \\
\left(\mu_{0} v\right)_{p, \Gamma}^{p} \leq c\|v\|_{W_{p}^{1-1 / p}\left(\Gamma_{0}\right)}^{p}, & p>n-1, \\
\left\|\mu_{0} v\right\|_{p, \Gamma}^{p} \leq c\|v\|_{W_{p}^{1-1 / p}\left(\Gamma_{0}\right)}^{p}, & p=n-1 .
\end{aligned}
$$

It is easily seen that for $p \in(1, \infty)$ the space $T W_{p}^{1}(G)$ is embedded in $T W_{p}^{1}(\Omega)$ continuously, so that (5.6) follows from Lemma 6. To prove (5.7), we observe that $|x-\xi| \sim|z-\zeta|$ for $|\zeta-z|>M(z, \zeta)$. Recalling (5.2), we dominate the left-hand side of (5.7) by $c I_{1}+c I_{2}$, where

$$
\begin{aligned}
& I_{1}=\int_{\Gamma} \frac{|v(x)|^{p}}{\varphi(z)^{n-2}} d s_{x} \int_{0}^{z-\varphi(z)} \frac{\left|\mu_{0}(z)-\mu_{0}(\zeta)\right|^{p}}{|z-\zeta|^{p+2-n}} d \zeta, \\
& I_{2}=\int_{\Gamma} \frac{d s_{x}}{\varphi(z)^{p}} \int_{\{\xi \in \Gamma: \zeta<z-\varphi(z)\}} \frac{\mu_{0}(\zeta)^{p}|v(x)-v(\xi)|^{p} d s_{\xi}}{\varphi(\zeta)^{n-2}} .
\end{aligned}
$$

Since $\mu_{0}(t)=0$ for $t \in\left(0, z_{1}\right)$, it follows that

$$
\begin{aligned}
& I_{1} \leq c \int_{\left\{x \in \Gamma: z>z_{1}\right\}}|v(x)|^{p} d s_{x} \int_{0}^{1}|z-\zeta|^{n-2} d \zeta \leq c\|v\|_{L_{p}\left(\Gamma_{0}\right)}^{p}, \\
& I_{2} \leq c \int_{\left\{x \in \Gamma: z>z_{1}\right\}} d s_{x} \int_{\left\{\xi \in \Gamma: \zeta>z_{1}\right\}}\left(|v(x)|^{p}+|v(\xi)|^{p}\right) d s_{\xi} \leq c\|v\|_{L_{p}\left(\Gamma_{0}\right)}^{p} .
\end{aligned}
$$

Hence, we obtain (5.7). 
The proof of (5.8) is similar. From (5.1) we deduce that the left-hand side of (5.8) is dominated by

$$
\begin{aligned}
c \int_{\left\{x \in \Gamma: z>z_{1}\right\}}|v(x)|^{p} d s_{x} & \int_{0}^{z-\varphi(z)} \frac{\left|\mu_{0}(z)-\mu_{0}(\zeta)\right|^{p}}{|z-\zeta|} d \zeta \\
& +c \int_{\left\{x \in \Gamma: z>z_{1}\right\}} d s_{x} \int_{\left\{\xi \in \Gamma: \zeta>z_{1}\right\}}\left(|v(x)|^{p}+|v(\xi)|^{p}\right) \mu_{0}(\zeta)^{p} d s_{\xi},
\end{aligned}
$$

which does not exceed $c\|v\|_{L_{p}\left(\Gamma_{0}\right)}^{p}$.

Lemma 9. Suppose $v \in T W_{p}^{1}(G)$ and $v$ has support in $\left\{x \in \Gamma: z \leq z_{0}\right\}$. Then

$$
\|v\|_{T W_{p}^{1}(G)}^{p} \leq c|v|_{p, \Gamma}^{p}+c \int_{\Gamma}|v(x)|^{p} \frac{d s_{x}}{\varphi(z)^{p-1}} .
$$

Proof. By Lemma 7, we have

$$
\|v\|_{W_{p}^{1-1 / p}\left(\Gamma_{0}\right)} \leq c|v|_{p, \Gamma}
$$

Therefore, (5.9) will be verified if we check the inequalities

$$
\begin{gathered}
(v)_{p, \Gamma}^{p} \leq c \int_{\Gamma}|v(x)|^{p} \frac{d s_{x}}{\varphi(z)^{p-1}}, \quad p>n-1, \\
\|v\|_{p, \Gamma}^{p} \leq c \int_{\Gamma}|v(x)|^{p} \frac{d s_{x}}{\varphi(z)^{p-1}}, \quad p=n-1,
\end{gathered}
$$

where $\|v\|_{p, \Gamma}$ and $(v)_{p, \Gamma}$ are the seminorms (5.1) and (5.2). We consider two symmetric cases where $z>\zeta$ and $z<\zeta$ separately and keep in mind that $|z-\zeta| \sim|x-\xi|$ for $|z-\zeta|>M(z, \zeta)$, obtaining

$$
\begin{aligned}
(v)_{p, \Gamma}^{p} & \leq c \int_{\Gamma} \frac{|v(x)|^{p} d s_{x}}{\varphi(z)^{n-2}} \int_{\{\xi \in \Gamma:|z-\zeta|>\varphi(z)\}} \frac{\varphi(\zeta)^{2-n} d s_{x}}{|z-\zeta|^{p+2-n}} \\
& \leq c \int_{\Gamma}|v(x)|^{p} \frac{d s_{x}}{\varphi(z)^{n-2}} \int_{|z-\zeta|>\varphi(z)} \frac{d \zeta}{|z-\zeta|^{p+2-n}} \leq c \int_{\Gamma}\left|v(x)^{p}\right| \frac{d s_{x}}{\varphi(z)^{p-1}} .
\end{aligned}
$$

For $p=n-1$ we have $\varphi(z) \sim \varphi(\zeta)$ for $2^{-1} \leq z \zeta^{-1} \leq 2$, whence

$$
\begin{aligned}
\|v\|_{p, \Gamma}^{p} & \leq c \int_{\Gamma} \frac{|v(x)|^{p}}{\varphi(z)^{2 p-2}} d s_{x} \int_{\{\xi \in \Gamma:|z-\zeta|>\varphi(z)\}} \frac{d s_{\xi}}{|z-\zeta|\left(1+\log \left|\frac{z-\zeta}{\varphi(z)}\right|\right)^{p}} \\
& \leq c \int_{\Gamma} \frac{|v(x)|^{p}}{\varphi(z)^{p-1}} d s_{x} \int_{|z-\zeta|>\varphi(z)} \frac{d \zeta}{|\zeta-z|\left(1+\log \left|\frac{\zeta-z}{\varphi(z)}\right|\right)^{p}} \\
& \leq c \int_{\Gamma}|v(x)|^{p} \frac{d s_{x}}{\varphi(z)^{p-1}} .
\end{aligned}
$$

The next assertion is similar to Lemma 3.

Lemma 10. Let $v \in T W_{p}^{1}(G)$. Then

$$
\|\bar{v}\|_{Y_{p}(0,1)} \leq c\|v\|_{T W_{p}^{1}(G)} .
$$

Proof. For $p \in(1, \infty)$, we write the norm on the left in (5.12) as follows:

$$
\begin{aligned}
& \|\bar{v}\|_{Y_{p}(0,1)} \\
& =\left(\int_{0}^{1} Q(z)|\bar{v}(z)|^{p} d z+|\bar{v}|_{p, \Gamma}^{p}+\int_{0}^{1} \int_{0}^{1} \sigma(z, \zeta)|\bar{v}(z)-\bar{v}(\zeta)|^{p} \frac{d z d \zeta}{|z-\zeta|^{p+2-n}}\right)^{1 / p}
\end{aligned}
$$


where

$$
|\bar{v}|_{p, \Gamma}^{p}=\int_{0}^{1} \int_{0}^{1}|u(z)-u(\zeta)|^{p} \frac{M(z, \zeta)^{n-2}}{|z-\zeta|^{p}} \chi\left(\frac{|z-\zeta|}{M(z, \zeta)}\right) d z d \zeta,
$$

$\chi$ is the characteristic function of the interval $(0,1)$, and $Q, \sigma$ are nonnegative weight functions,

$$
\begin{gathered}
Q(z)= \begin{cases}\varphi(z)^{n-1-p} & \text { if } p<n-1, \\
(\log (z / \varphi(z)))^{1-p} & \text { if } p=n-1, \\
1 & \text { if } p>n-1,\end{cases} \\
\sigma(z, \zeta)= \begin{cases}0 & \text { if } p<n-1, \\
\left(1+\log \left(\frac{|z-\zeta|}{M(z, \zeta)}\right)\right)^{-p} \chi_{(1 / 2,2)}\left(z \zeta^{-1}\right) \chi\left(\frac{M(z, \zeta)}{|z-\zeta|}\right) & \text { if } p=n-1, \\
\chi(M(z, \zeta) /|z-\zeta|) & \text { if } p>n-1 .\end{cases}
\end{gathered}
$$

Since $T W_{p}^{1}(G) \subset T W_{p}^{1}(\Omega)$, the inequality

$$
|\bar{v}|_{p, \Gamma} \leq c\|v\|_{T W_{p}^{1}(G)}
$$

follows from Lemma 3 .

Next, applying Hölder's inequality, we arrive at

$$
|\bar{v}(z)|^{p} \leq c \varphi(z)^{2-n} \int_{\gamma_{z}}|v(y, z)|^{p} d \gamma_{z}(y), \quad \gamma_{z}=\{y: y / \varphi(z) \in \gamma=\partial \omega\},
$$

whence

$$
\int_{0}^{1} Q(z)|\bar{v}(z)|^{p} d z \leq c \int_{\Gamma} \frac{Q(z)}{\varphi(z)^{n-2}}|v(x)|^{p} d s_{x}
$$

where $d s_{x}$ is the area element of $\Gamma$. If $p<n-1$, the required result follows from (5.14) and the last estimate.

For $p \geq n-1$ we must bound the last term in (5.13). Using Hölder's inequality once again to bound the mean value (3.1), we get

$$
|\bar{v}(z)-\bar{v}(\zeta)|^{p} \leq c \int_{\gamma}|v(\varphi(z) Y, z)-v(\varphi(\zeta) Y, \zeta)|^{p} d \gamma(Y) .
$$

Integration with respect to a fictitious variable $Y^{\prime} \in \gamma$ yields

$$
\begin{aligned}
|\bar{v}(z)-\bar{v}(\zeta)|^{p} \leq & c \iint_{\gamma \times \gamma}\left|v(\varphi(z) Y, z)-v\left(\varphi(z) Y^{\prime}, z\right)\right|^{p} d \gamma(Y) d \gamma\left(Y^{\prime}\right) \\
& +c \iint_{\gamma \times \gamma}\left|v\left(\varphi(z) Y^{\prime}, z\right)-v(\varphi(\zeta) Y, \zeta)\right|^{p} d \gamma(Y) d \gamma\left(Y^{\prime}\right) .
\end{aligned}
$$

Integrating with respect to $z, \zeta$, we arrive at the inequality

$$
\int_{0}^{1} \int_{0}^{1} \sigma(z, \zeta)|\bar{v}(z)-\bar{v}(\zeta)|^{p} \frac{d z d \zeta}{|z-\zeta|^{p+2-n}} \leq c I_{1}+c I_{2},
$$

where

$$
\begin{aligned}
& I_{1}=\iint_{\Gamma \times \Gamma} \frac{|v(x)-v(\xi)|^{p}}{|z-\zeta|^{p+2-n}}(\varphi(z) \varphi(\zeta))^{2-n} \sigma(z, \zeta) d s_{x} d s_{\xi} \\
& I_{2}=\int_{0}^{1} d z \iint_{\gamma \times \gamma}\left|v(\varphi(z) Y, z)-v\left(\varphi(z) Y^{\prime}, z\right)\right|^{p} d \gamma(Y) d \gamma\left(Y^{\prime}\right) \int_{0}^{1} \frac{\sigma(z, \zeta)}{|z-\zeta|^{p+2-n}} d \zeta .
\end{aligned}
$$

The definitions (5.1), (5.2) imply

$$
I_{1} \leq \begin{cases}c\|v\|_{p, \Gamma}^{p} & \text { if } p=n-1, \\ c(v)_{p, \Gamma}^{p} & \text { if } p>n-1 .\end{cases}
$$


Thus, the proof of (5.12) reduces to the proof of the estimate $I_{2} \leq c\|v\|_{T W_{p}^{1}(G)}^{p}$.

If $p>n-1$, then

$$
\int_{0}^{1} \frac{\sigma(z, \zeta) d \zeta}{|z-\zeta|^{p+2-n}} \leq c \int_{|\zeta-z|>\varphi(z)} \frac{d \zeta}{|z-\zeta|^{p+2-n}} \leq c \varphi(z)^{n-p-1}
$$

If $p=n-1$, we use the equivalence $\varphi(z) \sim \varphi(\zeta)$ for $2^{-1} \leq z \zeta^{-1} \leq 2$, obtaining

$$
\int_{0}^{1} \frac{\sigma(z, \zeta) d \zeta}{|z-\zeta|^{p+2-n}} \leq c \int_{|\zeta-z|>\varphi(z)} \frac{|\zeta-z|^{-1} d \zeta}{(1+\log (|\zeta-z| / \varphi(z)))^{p}} \leq c
$$

Hence, for $p \geq n-1$ we have

$$
\begin{aligned}
I_{2} & \leq c \int_{0}^{1} d z \iint_{\gamma_{z} \times \gamma_{z}}\left|v(y, z)-v\left(y^{\prime}, z\right)\right|^{p} \frac{d \gamma_{z}(y) d \gamma_{z}\left(y^{\prime}\right)}{\varphi(z)^{n+p-3}} \\
& \leq c \int_{0}^{1} d z \iint_{\gamma_{z} \times \gamma_{z}}\left|v(y, z)-v\left(y^{\prime}, z\right)\right|^{p} \frac{d \gamma_{z}(y) d \gamma_{z}\left(y^{\prime}\right)}{\left|y-y^{\prime}\right|^{n+p-3}},
\end{aligned}
$$

where $\gamma_{z}=\partial \omega_{z}, \omega_{z}=\left\{y \in \mathbb{R}^{n-1}: y / \varphi(z) \in \omega\right\}$. Since $T W_{p}^{1}(G)$ is embedded in $T W_{p}^{1}(\Omega)$, we can extend $v$ from the common boundary $\partial \Omega=\partial G$ up to a function $u \in W_{p}^{1}(\Omega)$ such that $\|u\|_{W_{p}^{1}(\Omega)} \leq c\|v\|_{T W_{p}^{1}(G)}$. For almost all $z \in(0,1)$ we have $u(\cdot, z) \in W_{p}^{1}\left(\omega_{z}\right)$, and the following estimate holds true by Gagliardo's theorem [1]:

$$
\iint_{\gamma_{z} \times \gamma_{z}}\left|v(y, z)-v\left(y^{\prime}, z\right)\right|^{p} \frac{d \gamma_{z}(y) d \gamma_{z}\left(y^{\prime}\right)}{\left|y-y^{\prime}\right|^{n+p-3}} \leq c\|\nabla u(\cdot, z)\|_{L_{p}\left(\omega_{z}\right)}^{p},
$$

where $c$ does not depend on $z, v$. Integrating the last inequality over $z \in(0,1)$, we find

$$
I_{2} \leq c\|\nabla u\|_{L_{p}(\Omega \cap U)}^{p} \leq c\|v\|_{T W_{p}^{1}(G)}^{p}
$$

and this completes the proof.

Proof of Theorem 3. (i) Remark 2 shows that $f^{(1)}=\mu_{0} f \in T W_{p}^{1}(\Omega)^{*}$. Next, we have

$$
\left|\left\langle f^{(1)}, v\right\rangle\right|=\left|\left\langle f, \mu_{0} v\right\rangle\right| \leq c\|f\|_{T W_{p}^{1}(G)^{*}}\left\|\mu_{0} v\right\|_{T W_{p}^{1}(G)} .
$$

By Lemma 8, the last-written norm does not exceed the quantity $c\|v\|_{W_{p}^{1-1 / p}\left(\Gamma_{0}\right)}$; thus, $f^{(1)} \in W_{p^{\prime}}^{-1 / p^{\prime}}\left(\Gamma_{0}\right)$.

Turning to (5.3), we note that

$$
\left\|\left(1-\mu_{0}\right) \bar{v}\right\|_{T W_{p}^{1}(G)} \sim\left\|\left(1-\mu_{0}\right) \bar{v}\right\|_{Y_{p}(0,1)},
$$

whence

$$
\left|\left\langle f^{(2)}, v\right\rangle\right| \leq c(f)\left\|\left(1-\mu_{0}\right) \bar{v}\right\|_{Y_{p}(0,1)} .
$$

Thus, (5.3) is a consequence of the estimate

$$
\left\|\left(1-\mu_{0}\right) \bar{v}\right\|_{Y_{p}(0,1)} \leq c\|\bar{v}\|_{Y_{p}(0,1)} .
$$

For $p<n-1$, the proof of (5.15) repeats the corresponding argument of Theorem 2 almost word for word. Let $p>n-1$. Then

$$
\begin{aligned}
\left\|\left(1-\mu_{0}\right) \bar{v}\right\|_{Y_{p}(0,1)}^{p} \leq & \int_{0}^{1}\left|\left(1-\mu_{0}(z)\right) \bar{v}(z)\right|^{p} d z \\
& +\int_{0}^{1} \int_{0}^{1} \frac{\left|\left(1-\mu_{0}(z)\right) \bar{v}(z)-\left(1-\mu_{0}(\zeta)\right) \bar{v}(\zeta)\right|^{p}}{|z-\zeta|^{p+2-n}} \sigma(z, \zeta) d z d \zeta
\end{aligned}
$$

where

$$
\sigma(z, \zeta)=\max \left\{\frac{M(z, \zeta)^{n-2}}{|z-\zeta|^{n-2}}, 1\right\}
$$


Clearly the right-hand side of (5.16) is not greater than

$$
c\|\bar{v}\|_{Y_{p}(0,1)}^{p}+c \int_{0}^{1} \int_{0}^{1}|\bar{v}(z)|^{p} \max \left\{M(z, \zeta)^{n-2},|z-\zeta|^{n-2}\right\} d z d \zeta .
$$

The integral over $(0,1) \times(0,1)$ does not exceed $\|\bar{v}\|_{L_{p}(0,1)}^{p}$, and (5.15) follows.

If $p=n-1$, we have

$$
\left\|\left(1-\mu_{0}\right) \bar{v}\right\|_{Y_{p}(0,1)}^{p} \leq c\|\bar{v}\|_{Y_{p}(0,1)}^{p}+c \int_{0}^{1} \int_{0}^{1} \frac{\left|\mu_{0}(z)-\mu_{0}(\zeta)\right|^{p}|\bar{v}(z)|^{p}}{|z-\zeta|} \sigma(z, \zeta) d z d \zeta,
$$

where the weight $\sigma$ does not vanish only for $2^{-1} \leq z \zeta^{-1} \leq 2$ and satisfies $\sigma(z, \zeta)|z-\zeta|^{p-1} \leq$ const. Since $\left.\mu_{0}\right|_{\left(0, z_{1}\right)}=0$, the last integral over $(0,1) \times(0,1)$ is not greater than

$$
c \int_{z_{1} / 2}^{1}|\bar{v}(z)|^{p} d z \int_{0}^{1}|z-\zeta|^{p-1} \sigma(z, \zeta) d \zeta,
$$

which does not exceed $c \int_{z_{1} / 2}^{1}|\bar{v}(z)|^{p} d z$, and therefore, is not greater than $c\|\bar{v}\|_{Y_{p}(0,1)}^{p}$. Hence (5.15) is proved also for $p=n-1$. Thus, (5.3) is established. Furthermore, the continuity of the functional

$$
T W_{p}^{1}(G) \ni v \mapsto\left\langle\left(1-\mu_{0}\right) \bar{f}, v\right\rangle
$$

is a consequence of Lemma 10 .

We turn to (5.4). Arguing in the same way as in the proof of inequality (4.2) in Theorem 2, we arrive at estimate (4.10), which can be rewritten in the form

$$
\sum_{k=1}^{N} \alpha_{k}^{p^{\prime}} \leq 2\left\langle\left(1-\mu_{0}\right)(f-\bar{f}), v\right\rangle,
$$

where

$$
v(x)=\sum_{k=1}^{N} \alpha_{k}^{p^{\prime}-1} \nu_{k}(z)\left(v_{k}(x)-\stackrel{\circ}{v}_{k}\right), \quad x \in \partial \Omega,
$$

$\nu_{k}(z) v_{k}(x)=0$ outside $\Gamma_{k}$, and the notation is the same as in Theorem 2. To bound $\|v\|_{T W_{p}^{1}(G)}$, we note that $v(x)=0$ outside the set $\left\{x \in \Gamma: z \leq z_{0}\right\}$, so that (5.9) is valid.

Estimating the seminorm $|v|_{p, \Gamma}$ in the same way as in Theorem 2, we arrive at

$$
|v|_{p, \Gamma}^{p} \leq c \sum_{k=1}^{N} \alpha_{k}^{p^{\prime}}
$$

To bound the last term in (5.9), we can write

$$
\begin{aligned}
\int_{\Gamma}|v(x)|^{p} \frac{d s_{x}}{\varphi(z)^{p-1}} & \leq c \sum_{k=1}^{N} \alpha_{k}^{\left(p^{\prime}-1\right) p} \int_{\Gamma} \nu_{k}(z)^{p}\left|v_{k}(x)-\stackrel{\circ}{v}_{k}\right|^{p} \frac{d s_{x}}{\varphi(z)^{p-1}} \\
& \leq c \sum_{k=1}^{N} \alpha_{k}^{p^{\prime}} \varphi\left(z_{k}\right)^{1-p}\left\|v_{k}-\stackrel{\circ}{v}_{k}\right\|_{L_{p}\left(\Gamma_{k}\right)}^{p} \leq c \sum_{k=1}^{N} \alpha_{k}^{p^{\prime}}\left[v_{k}\right]_{p, \Gamma_{k}}^{p}
\end{aligned}
$$


(we have used Lemma 5 at the last step). Since $\left[v_{k}\right]_{p, \Gamma_{k}} \leq 1$ by assumption, we get

$$
\int_{\Gamma}|v(x)|^{p} \frac{d s_{x}}{\varphi(z)^{p-1}} \leq c \sum_{k=1}^{N} \alpha_{k}^{p^{\prime}} .
$$

Combining (5.9) and (5.18)-(5.19) yields

$$
\|v\|_{T W_{p}^{1}(G)}^{p} \leq c \sum_{k=1}^{N} \alpha_{k}^{p^{\prime}} .
$$

This inequality and (5.17) lead to the estimate

$$
\sum_{k=1}^{N} \alpha_{k}^{p^{\prime}} \leq c\left\|\left(1-\mu_{0}\right)(f-\bar{f})\right\|_{T W_{p}^{1}(G)^{*}}\left(\sum_{k=1}^{N} \alpha_{k}^{p^{\prime}}\right)^{1 / p},
$$

and we arrive at (5.4).

(ii) If $h \in W_{p^{\prime}}^{-1 / p^{\prime}}\left(\Gamma_{0}\right)$, then $f^{(1)}=\mu_{0} h \in W_{p^{\prime}}^{-1 / p^{\prime}}\left(\Gamma_{0}\right)$ by the Gagliardo theorem and Remark 2. Moreover, since $T W_{p}^{1}(G) \subset W_{p}^{1-1 / p}\left(\Gamma_{0}\right)$, it follows that $f^{(1)} \in T W_{p}^{1}(G)^{*}$.

Under the hypotheses of Theorem $3, f^{(3)}$ is continuous on $T W_{p}^{1}(\Omega)$ by Theorem 2 . Therefore, $f^{(3)}$ is also continuous on $T W_{p}^{1}(G)$, because $T W_{p}^{1}(G) \subset T W_{p}^{1}(\Omega)$. This fact and (4.5) imply (5.5).

In conclusion, we verify the continuity of the functional $T W_{p}^{1}(G) \ni v \mapsto\left\langle f^{(2)}, v\right\rangle$. Since $g \in Y_{p}(0,1)^{*}$, we have

$$
\left|\left\langle f^{(2)}, v\right\rangle\right| \leq c(g)\left\|\left(1-\mu_{0}\right) \bar{v}\right\|_{Y_{p}(0,1)} .
$$

Now the required result follows from (5.15) and Lemma 10. The proof is complete.

\section{REFERENCES}

[1] E. Gagliardo, Caratterizzazioni delle tracce sulla frontiera relative ad alcune classi di funzioni in $n$ variabili, Rend. Sem. Mat. Univ. Padova 27 (1957), 284-305. MR0102739 (21:1525)

[2] J. Leray and J.-L. Lions, Quelques résultats de Višik sur les problèmes elliptiques non linéaires par les méthodes de Minty-Browder, Bull. Soc. Math. France 93 (1965), 97-107. MR0194733 (33:2939)

[3] S. L. Sobolev, Some applications of functional analysis in mathematical physics, Leningrad. Gos. Univ., Leningrad, 1950; English transl. from 3rd Russian ed., Transl. Math. Monogr., vol. 90, Amer. Math. Soc., Providence, RI, 1991. MR0052039 (14:565a), MR1125990 (92e:46067)

[4] V. G. Maz'ya, Functions with a finite Dirichlet integral in a domain with a cusp at the boundary, Zap. Nauchn. Sem. Leningrad. Otdel. Mat. Inst. Steklov. (LOMI) 126 (1983), 117-137; English transl. in J. Soviet Math. 27 (1984), no. 1. MR0697431 (84k:46030)

[5] _ Sobolev spaces, Leningrad. Univ., Leningrad, 1985; English transl., Springer-Verlag, Berlin, 1985. MR0807364 (87g:46055) MR0817985 (87g:46056)

[6] V. P. Glushko, On domains which are star-like relative to a sphere, Dokl. Akad. Nauk SSSR 144 (1962), no. 6, 1215-1216; English transl. in Soviet Math. Dokl. 3 (1962). MR0141976 (25:5371)

[7] V. G. Maz'ya and S. V. Poborchǐ̌, Traces of functions in Sobolev spaces on a boundary of a domain with a cusp, Trudy Inst. Mat. (Novosibirsk) 14 (1989), 182-208; English transl., Siberian Adv. Math. 1 (1991), no. 3, 75-107. MR.1040488 (91e:46042) MR.1128379

[8] - Imbedding and continuation theorems for functions in non-Lipschitz domains, S.Peterburg. Gos. Univ., St. Petersburg, 2006, 399 pp. (Russian)

[9] E. M. Stein, Singular integrals and differentiability properties of functions, Princeton Math. Ser., No. 30, Princeton Univ. Press, Princeton, NJ, 1970. MR0290095 (44:7280)

[10] S. V. Poborchiı̌, Continuity of the boundary trace operator $W_{p}^{1}(\Omega) \rightarrow L_{q}(\partial \Omega)$ for a domain with outward peak, Vestnik S.-Peterburg. Univ. Ser. 1 2005, vyp. 3, 51-60; English transl., Vestnik St. Petersburg Univ. Math. 38 (2005), no. 3, 37-44 (2006). MR2229350 (2006m:35063) 
[11] O. V. Besov, V. P. Il'in, and S. M. Nikol'skiu, Integral representations of functions, and imbedding theorems, "Nauka", Moscow, 1996; English transl. of 1st ed., Vol. I, II, V. H. Winston and Sons, Washington, DC, Vol. I, 1978; Vol. II, 1979. MR1450401 (98b:46037) MR0519341 (80f:46030a) MR 0521808 (80f:46030b)

[12] P. W. Jones, Quasiconformal mappings and extendability of functions in Sobolev spaces, Acta Math. 147 (1981), no. 1-2, 71-88. MR0631089 (83i:30014)

Department of Mathematics, Linköping University, 58183 Linköping, Sweden

E-mail address: vlmaz@mai.liu.se

Department of Mathematics and Mechanics, St. Petersburg State University, Universitetskil Prospekt 28, Petrodvorets, 198504 St. Petersburg, Russia

E-mail address: Sergei.Poborchi@paloma.spbu.ru

Received 14/JAN/2008

Translated by S. V. POBORCHII 